\title{
APLICACIÓN YCALIBRACIÓNDE UNA INTEGRACIÓN DE LOS MODELOS TREX Y SWMM PARA LA SIMULACIÓN HIDROLÓGICA-HIDRÁULICA DISTRIBUIDA EN UNA CUENCA FUERTEMENTE ANTROPIZADA
}

\section{APPLICATION AND CALIBRATION OF AN INTEGRATION OF THE TREX AND SWMM MODELS FOR DISTRIBUTED HYDROLOGICAL-HYDRAULIC SIMULATION IN A STRONGLY ANTHROPIZED BASIN}

\author{
Pablo TomásStehli( ${ }^{(1)(*)}$, Juan FranciscoWeber ${ }^{(1)}$ y Leandro Redin Vestena $^{(2)}$ \\ (1) Facultad Regional Córdoba. Universidad Tecnológica Nacional. Córdoba, Argentina. \\ (2) Departamento de Geografía, Universidade Estadual do Centro-Oeste, Guarapuava, Brasil. \\ ${ }^{(*)}$ e-mail: pablo_stehli@hotmail.com
}

\begin{abstract}
RESUMEN
Este trabajo describe la aplicación y calibración de un modelo hidrológico espacialmente distribuido en la cuenca del Rio Cascavel, en el estado de Paraná, Brasil. Se aplicó el modelo TREX incluyendo una modificación en su código fuente que permite la simulación de bocas de tormenta; y en conjunto se aplicó el modelo hidrodinámico semidistribuido SWMM para simular los flujos en los conductos y cauces.La recopilación y procesamiento de información incluyó: la corrección del modelo digital de elevación,mapas de tipos y usos de suelo, relevamiento de la ubicación de bocas de tormenta y conductos. Finalmente, se seleccionaron una serie de eventos (precipitaciones y caudales líquidos medidos) para la calibración del modelo para la cuenca de estudio. En el trabajo se presentan los resultados de la calibración de cinco eventos seleccionados, obteniendo un aceptable ajuste del modelo. Se concluyó de esta forma, que los modelos utilizados en su conjunto permiten la simulación completa de una cuenca urbana. En particular, las características de TREX, permite utilizar información raster, agilizando el ingreso de datos para la modelación totalmente distribuida de una cuenca urbana.
\end{abstract}

Palabras Claves: Modelación hidrológica, modelación hidráulica, SWMM,TREX.

\begin{abstract}
This paper is about the application and calibration of a spatially distributed model in the watershed of Cascabel river, in the state of Parana, Brazil. For this reason, first a TREX model previously adaptated is used to model elements of the urban watersheds, sucha as storminlets, and then the semidistributed SWMN model is used to simulate the flow in the conducts and channels. The basin data were collected and processed to carry out these works, as for example the correction and adaptation of the digital elevation model, the maps about the land types and its uses, the survey about the location of the storm inlets and conducts. Finally, a series of events were selected (pairs of measured rainfall and liquid flow data) to perform the calibration of the model for the basin studied. The work presents the results of the calibration of five selected events, obtaining an acceptable fit of the model. In this way, it was concluded that the models used as a whole allow the complete simulation of an urban basin. In particular, the characteristics of TREX allow the use of raster information, speeding up data entry for the fully distributed modeling of an urban watershed.
\end{abstract}

KEYWORDS: Hydrological modeling,hydraulic modeling,SWMM,TREX. 


\section{INTRODUCCIÓN}

Los entornos urbanos son sistemas muy complejos debido a su variabilidad en una amplia gama de escalas espaciotemporales, y a la interacción entre las actividades humanas y los procesos naturales. Un ejemplo de ello, es el continuo proceso de urbanización que cambia la cobertura de la tierra y ejerce una fuerte influencia en el comportamiento hidrológico de las cuencas urbanas.

A lo largo del tiempo, se han desarrollado diversos modelos hidrológicos para ser aplicados en cuencas urbanas. Los mismos se pueden clasificar como de base física, conceptual o empírica, pero también se pueden clasificar según su resolución espacial y su capacidad de representar la complejidad de la hidrología urbana, de esta forma se agrupan en semidistribuidos, totalmente distribuidos o de unidades de respuestas hidrológica (HRU).

En este trabajo se utiliza un modelo hidrológico totalmente distribuido como TREX para modelar los procesos hidrológicos superficiales que se ocasionan en una cuenca urbana y SWMM para simular los procesos hidráulicos que allí se producen.

Se presenta la aplicación de ambos modelos en conjunto en una cuenca urbana y la calibración de los parámetros de los modelos para cinco eventos.

Un modelo de este tipo hidrológico-hidráulico totalmente distribuido calibrado para una cuenca fuertemente urbanizada podrá ser una herramienta más para la gestión de los desagües pluviales urbanos como ser el análisis del impacto de nuevas urbanizaciones, cambios de uso de suelo en la cuenca y capacidad de elementos hidráulicos entre otras aplicaciones.

\section{OBJETIVOS}

Como objetivo general se puede mencionar el de desarrollar y validar una metodología para la modelación distribuida de escurrimiento superficial en áreas urbanas, en base al formato raster de información.Sumado a esto, se fijaron los siguientes objetivos particulares:

Aplicar el modelo hidrológico distribuido TREX (modelo raster) a la cuenca del Río Cascavel para simular eventos históricos de interés, calibrando sus parámetros para lograr la mejor representación de la respuesta hidrológica observada.

Utilizar el modelo SWMM para la modelación de los conductos y canalizaciones y la cuenca del Río Cascavel.

\section{REVISIÓN BIBLIOGRÁFICA}

Para evaluar la respuesta hidrológica de un sistema, regularmente es indispensable la elaboración de algún tipo de esquema, por medio del cual se pueda representar en forma simplificada, un sistema real; en otras palabras: un modelo. El mismo podrá ser utilizado para reconstruir eventos pasados y predecir los futuros (Puricelli, 2003).

El modelo es la representación de algún objeto o sistema, en un lenguaje o forma de fácil acceso y uso, con el objetivo de entenderlo y poder generar sus respuestas para diferentes entradas. Un modelo hidrológico es una herramienta que la ciencia desarrolló para entender mejor y representar el comportamiento de la cuenca hidrográfica y prever condiciones diferentes a las observadas (Tucci, 1998).

Los modelos hidrológicos se empiezan a desarrollar en los años 1960. En 1972 comienzan a publicarse los primeros estudios de efectos de urbanización sobre cuencas. Posteriormente se desarrollan modelos que incluían la simulación de embalses de detención. Se desarrollan modelos como el ILLUDAS (Universidad de Illinois), el HEC-1 (Centro de Ingeniería Hidrológica), el TR55 (Servicio de Conservación de Suelos), STORM (Cuerpo de Ingenieros EEUU). Además surge un poderoso modelo como el SWMM (EPA Agencia norteamericana de protección del Medio Ambiente) que incorpora el transporte de contaminantes (Riccardi, 1997).

En Argentina se han desarrollado modelos como OCINE-2 (UNL, Santa Fe, Argentina, 1986); ARHYMO (CRA. INCYTH. Argentina, 1994); los que son versiones modificadas del modelo OCINE en el caso del primero y del HYMO10 y OTTHYMO el segundo (Riccardi, 1997).

Luego, en los párrafos siguientes se describirá en primer lugar las clasificaciones típicas de los modelos hidrológicos, especialmente en función de su representación espacial y luego más en detalle los modelos hidrológicos utilizados actualmente en el ámbito urbano. 
Los modelos, se pueden clasificar según la naturaleza de los algoritmos empleados (empíricos, conceptuales o con base física; Salvadore et al., 2015), o su resolución espacial y cómo representan la complejidad del programa de hidrología urbana.

Los modelos agregados (Kleidorfer et al., 2009) y semi-distribuidos (INSA-Valor, 1999) son modelos conceptuales y dependen de una representación simplificada de la heterogeneidad de la cuenca urbana. De hecho, toda la cuenca se considera como una sola unidad con características homogéneas para los agregados, mientras que la cuenca se divide en un número limitado de homogéneas subcuencas para los modelos semidistribuidos. Estos dos enfoques fueron ampliamente desarrollados y utilizados porque requieren una cantidad limitada de datos para su implementación, y muestran un tiempo de cálculo rápido. A menudo, en el proceso de calibración, estos modelos de "fuerzan" para representar los datos observados. Sin embargo, estos modelos dan información de salida en la escala de subcuencas, que puede ser una escala muy grande como para cumplir con los requisitos de la gestión de las aguas urbanas para entender algunos problemas de inundación muy local o para evaluar estrategias de manejo a escalas muy pequeñas.

Los modelos hidrológicos totalmente distribuidos son los más complejos porque tienen en cuenta la heterogeneidad espacial en las entradas y los parámetros. Éstos, separan los procesos a simular en elementos pequeños o celdas de cuadrícula. También pueden estar estructurados como un modelo basado en la física que los hace más identificables con el proceso hidrológico real. Los modelos espacialmente distribuidos han influido en las prácticas de gestión al proporcionar datos detallados para elementos pequeños (Sitterson, et al., 2017).

Los modelos que se describen a continuación son utilizados en el ámbito urbano. Los mismos fueron seleccionados a partir de una serie de trabajos que realizaron una recopilación o comparación de modelos hidrológicos utilizados en ámbitos urbanos con distintos objetivos (Abdullah et al., 2019; Lind, 2015; Rangari et al., 2015; Armitage et al., 2014; Ochoa-Rodriguez et al., 2013; Mitchell et al., 2007; Freni et al, 2003). La información se amplió con recopilación propia y con información de los manuales (cuando se encuentran disponibles) de cada uno de los modelos.

El programa SWMM (Storm Water Management
Model) fue elaborado por la USEPA (U.S. Environmental Protection Agency) (Rossman, 2010).Es un modelo ampliamente difundido en áreas urbanas, pero que en realidad es un modelo semidistribuido, donde las cuencas son unidades hidrológicas de terreno cuya topografía y elementos del sistema de drenaje conducen la escorrentía directamente hacia un punto de descarga. El usuario del programa es el encargado de dividir el área de estudio en el número adecuado de cuencas e identificar el punto de salida de cada una de ellas. Los puntos de salida de cada una de las cuencas pueden ser bien nudos del sistema de drenaje o bien otras cuencas.

PCSWMM es una interfaz para SWMM y está desarrollada por Computational Hydraulics Inc. (CHI) (https://www.pcswmm.com/). Es un modelo integrado de SIG que utiliza SWMM 5.0 como modelo de motor computacional para los cálculos hidrológicos e hidráulicos. Es una herramienta de modelado independiente con todas las herramientas SIG necesarias incluidas y tiene soporte para varios formatos CAD y GIS (Shamsi, 2005; CHI, 2014b, citados en Lind, 2015). Los modelos PCSWMM se pueden usar tanto para predicciones continuas a largo plazo como para modelado de eventos únicos. Es un modelo de captación integrada que incluye alcantarillas pluviales, alcantarillas combinadas, sistemas fluviales, plantas de tratamiento y aguas receptoras. El área de captación se puede dividir en subcuencas y el modelo se puede utilizar para cálculos 1D y 2D (Lind, 2015).

XP Storm y XP SWMM son productos que actualmente se encuentran bajo licencia del grupo de investigación "HR Wallington Software", igual que Infoworks. Es posible obtener información de los modelos a partir del sitio web de dicho grupo (https://www.innovyze.com/en-us/products/xpstormy https://www.innovyze.com/

us/products/xpswmm). XPStorm y XPSWMM originalmente fueron desarrollados por XP Solutions de Australia.

XPStorm es un modelo hidráulico e hidrológico integrado utilizado para sistemas de aguas pluviales y fluviales/gestión de llanuras de inundación. Las áreas de uso en relación con el análisis, diseño y planificación de aguas pluviales incluyen, entre otros, el diseño del plan maestro de aguas pluviales, estructuras de bajo impacto de escurrimiento, optimización de estanques de detención, inundaciones urbanas $1 \mathrm{D}$ y $2 \mathrm{D}$. El programa tiene integraciones 
SIG y CAD y puede importar y exportar varios formatos SIG y CAD. Los formatos SWMM 5.0 también se pueden importar y exportar. Los resultados de la simulación pueden presentarse como documentos de resultados, tablas, diagramas de perfil, mapeo de inundaciones y animaciones (Lind, 2015).

Al igual que XP Storm, XPSWMM incluye aguas pluviales y sistemas fluviales / llanuras aluviales. Además, también incluye la gestión de aguas residuales. El modelo simula flujos de red $1 \mathrm{D}$ en combinación con flujos terrestres 2D, estructuras LID y calidad de aguas pluviales. La herramienta se puede utilizar para sistemas naturales como, por ejemplo, estanques, ríos y lagos y entornos artificiales como tuberías, conductos y calles.

InfoWorks ofrece un modelado rápido, preciso y estable de los elementos clave de las aguas residuales y sistemas de alcantarillado. Ofrece una completa solución para el problema del modelado de los efectos de remanso y el flujo inverso, canales abiertos, alcantarillas maestras, conexiones de tuberías complejas y estructuras auxiliares.

InfoWorks CS-2D es un paquete de software que incluye un módulo para la simulación 2D del flujo terrestre utilizando una malla triangular. Por lo tanto, InfoWorks CS-2D permite el modelado de drenaje doble 1D-2D, con la interacción entre el alcantarillado y los sistemas de superficie que se producen en pozos de registro y barrancos. Cuando se utiliza InfoWorks CS-2D, es posible aplicar lluvia a través de subcuencas o directamente en el modelo 2D de la superficie. Sin embargo, este paquete de software no permite el modelado de lluvia-escorrentía cuando la lluvia se aplica directamente en el modelo 2D de la superficie. Para sortear este problema, el modelista debe calcular la escorrentía externamente y restarla del hietograma de lluvia antes de aplicarlo al modelo. Este problema se ha resuelto en el nuevo paquete de software InfoWorks ICM (Integrated Catchment Modeling), que constituye una actualización sobre InfoWorks CS y, entre otras mejoras, permite la estimación de la escorrentía cuando la lluvia se aplica directamente en el modelo 2D de la superficie (Ochoa-Rodriguez et al., 2013).

MIKE Urban es un paquete de software desarrollado por DHI y basado en SWMM 5.0. Es una herramienta de modelado completa integrada con SIG desarrollada por el grupo DHI, organización independiente de investigación y consultoría. El software MIKE URBAN se puede utilizar para sistemas de aguas pluviales y residuales, alcantarillas combinadas y separadas, así como sistemas de distribución de agua.

MIKE Urban es un software de módulos. El Model Manager es el módulo principal de MIKE Urban e incluye un módulo de datos común para los sistemas de distribución y recolección de agua. Es posible cargar archivos de SWMM5 y EPANET y archivos de resultados de cualquier tamaño. El motor SWMM5 se incluye para el modelado de aguas pluviales y alcantarillado, mientras que EPANET se incluye para el modelado de distribución de agua. El sistema de recolección consta de varios módulos adicionales como CS-Pipeflow, CSRainfall Runoff, CS- Pollutant Transport, CS- Control y CS- procesos biológicos. Para el módulo adicional CS-Pipeflow se utiliza el motor computacional MOUSE (Modelado de alcantarillas urbanas).a largo plazo, a través de estadísticas.

Los módulos anteriormente citados se combinan y se acoplan de distinta manera para poder modelar correctamente según el caso de estudio y sus necesidades. De esta forma es posible simular las precipitaciones, la evaporación (para un modelo continuo), el humedecimiento, la infiltración (mediante la relación de Horton), el almacenamiento superficial, escurrimiento superficial y el flujo en tuberías.

Modelo HYDRAS. Mobayed y Cruickshank (1998), Mobayed y Ortiz (2000) y Mobayed (2009) desarrollaron el modelo hidrológico distribuido HIDRAS, el cual integra bajo una misma plataforma el procesamiento de la información digital climatológica, hidrométrica, fisiográfica y topográfica para la estimación de parámetros hidrológicos y tiene implementados algoritmos de cálculo de las diferentes etapas del proceso lluvia-escurrimiento.

El modelo hidrológico distribuido HIDRAS usa la información de los modelos digitales de elevación (MDE), no sólo para definir direcciones de flujo y la acumulación de elementos para configurar la red de drenaje, sino también para hacer el escalado de la cuenca en celdas geomorfológicas, esto es una agrupación mínima y suficiente de elementos que dan forma a una superficie tributaria. Tal agrupación corresponde al área mínima capaz de formar un cauce incipiente de escurrimiento.

Modelo Casc-2d-TREX. El punto de partida para el desarrollo de TREX (Two-dimensional Runoff, Erosion, and eXport model) fue el modelo hidrológico 
CASC2D (CASCade of planes, 2-Dimensional (Julien\& Saghafian, 1991). La estructura básica es un modelo basado en eventos que simula el flujo superficial, la erosión de la superficie del suelo y la deposición, flujo en canal y transporte de sedimentos a través de los cauces fluviales (England et al., 2007). Como parte del desarrollo de TREX, los componentes hidrológicos y de transporte de sedimentos de CASC2D se ampliaron de manera significativa y mejoraron para soportar el modelado de las inundaciones y las características químicas de transporte (Velleux et al., 2008).

CASC2D y TREX fueron pensados originalmente para cuencas rurales pero se encuentran varios trabajos en los cuales se aplican en cuencas urbanas con aceptables resultados. El inconveniente se presenta principalmente porque no modela los flujos en tuberías. TREX y CASC2D son modelos de dominio público desarrollados por la Universidad Estatal de Colorado ubicada en Fort Collins (Colorado State University-Fort Collins), Estados Unidos.

Multi-Hydro, es un modelo totalmente distribuido, que se está desarrollando actualmente en la Ecole des Ponts ParisTech. Presenta varios componentes que interactúan entre sí para simular los distintos procesos hidrológicos e hidráulicos que intervienen en una cuenca periurbana. Cada componente se basa en modelos existentes y ampliamente validados, y que son software de dominio público. Los procesos modelados son las precipitaciones, la escorrentía, la infiltración en suelos heterogéneos, y el drenaje en los sistemas de alcantarillado (Giangola-Murzyn, 2013).

El modelado de los procesos de superficie está realizado por TREX (citado anteriormente). Con este modelo se realiza la simulación de los procesos superficiales: precipitación, intercepción, almacenamiento, infiltración y escorrentía superficial.

El modelado del flujo en zona no saturada se realiza con el software VS2DT (Healy, 1990). Este modelo permite simular el flujo y transporte en medios porosos variablementeen una y dos dimensiones. El modelo VS2DT es un modelo de diferencias finitas que resuelve la ecuación de Richards para el flujo y la ecuación de advección-dispersión para el transporte de solutos.

Por último para la simulación de las redes de saneamiento se utiliza el modelo SWMM (también ya descrito anteriormente).
El modelo Itzï es el trabajo del proyecto de Doctorado de Laurent Courty bajo la supervisión del Dr. Adrián Pedrozo-Acuña, de la Universidad Autónoma de México. Dicho proyecto se encuentra en desarrollo y es posible encontrar los avances del mismo, como así también tutoriales, ayudas, aplicaciones, etc. en la página web del proyecto: https://www.itzi.org/.

Itzï es un modelo hidrológico dinámico, totalmente distribuido que simula los flujos 2D de la superficie regular con la ayuda de mapa raster y el flujo en la red de drenaje a través del modelo SWMM.

El modelo Itzï, resuelve las ecuaciones de inercia parcial amortiguada, está escrito en lenguaje Python y es compatible con plataformas de Sistemas de Información Geográfica de libre distribución (por ejemplo GRASS) (Courty et al, 2017).

Itzï integra el modelo SWMM con un modelo inercial de superficie. Las interacciones entre el modelo de superficie y la red de drenaje se realizan en el nodo de la red a través de las ecuaciones de orificio, según el tirante de agua en cada modelo. Los dos modelos se ejecutan simultáneamente y el intercambio entre los dos son bidireccionales. El agua puede entrar o salir de la red de drenaje.

El modelo presentado resuelve las ecuaciones de aguas someras de forma simplificada, a través del método de diferencias finitas. El cálculo de flujo está compuesto por ecuaciones cuasi-bidimensionales, que resuelven de manera independiente el flujo en cada dimensión (Courty et al, 2017).

StormCAD es un producto de Bentley (https://www.bentley.com/es/products/product-

line/hydraulics-and-hydrology-software/stormcad) y es una herramienta utilizada para diseñar y analizar sistemas de alcantarillado de aguas pluviales. StormCAD utiliza el método racional para calcular los flujos máximos y no tiene en cuenta las estructuras de detención y los cambios de flujo a lo largo del tiempo (Lind, 2015).

Otro producto de Bentley es CivilStorm (https://www.bentley.com/es/products/product-

line/hydraulics-and-hydrology-software/civilstorm). Es un software de modelado de aguas pluviales que modela más aspectos del sistema que StormCAD. CivilStorm es un modelo dinámico que tiene en cuenta el almacenamiento, la detención y los flujos a lo largo del tiempo y, por lo tanto, es una herramienta de modelado más avanzada que 
StormCAD. Se utiliza para la planificación maestra y estudiar la calidad del agua. Puede, como StormCAD, usarse como un producto independiente pero también dentro de AutoCAD y otras herramientas de software. El usuario puede optar por utilizar el motor SWMM o la solución incorporada CivilStorms. Los resultados se pueden presentar como, por ejemplo, mapeo temático, gráficos dinámicos, perfiles y tablas (Lind, 2015).

El Modelo para la conceptualización de la mejora de las aguas pluviales urbanas (MUSIC) es una herramienta de evaluación de la calidad de las aguas pluviales desarrollada por la empresa australiana de gestión del agua, eWater. El modelo se utiliza para analizar los diseños conceptuales de la infraestructura de aguas pluviales y pone un énfasis particular en los objetivos de calidad del agua (Elliott y Trowsdale, 2007, citado en Armitage et al., 2014). MUSIC modela el control del flujo aguas abajo y los beneficios de calidad del agua logrados a través de la instalación de mejores prácticas de gestión (BMP) estructurales (Lloyd et al., 2002, citado en Armitage et al., 2014). Desarrollado por primera vez en 2001, el software está diseñado para ayudar a los profesionales urbanos de aguas pluviales a crear y visualizar estrategias para abordar los problemas asociados con la hidrología de las aguas pluviales y los impactos de la contaminación.

MUSIC puede operar en un rango de escalas espaciales y temporales; Puede simular cuencas de 0.01 a $100 \mathrm{~km}^{2}$ con pasos de tiempo que van desde 6 minutos a 24 horas.

La última versión de MUSIC, es decir, MUSIC 6.3 se lanzó en Noviembre de 2017, es posible su descarga en la página web australiana de eWater (https://ewater.org.au/products/music/).

Sobek fue desarrollado por la compañía holandesa Deltares y, como tal, es el software más utilizado en los Países Bajos.

Sobek es un paquete comercial para modelar procesos hidrológicos e hidráulicos tanto en áreas rurales como urbanas. Comprende una serie de módulos para aplicaciones específicas; uno de ellos es Sobek-Urban, que puede usarse para modelar sistemas de drenaje urbano e inundaciones pluviales urbanas. Sobek-Urban ofrece capacidades de modelado 1D y 2D. Al igual que InfoWorks CS, Sobek-Urban-1D es un paquete de modelado semidistribuido, con precipitaciones aplicadas al modelo a través de subcuencas, cada una de las cuales puede comprender diferentes tipos de superficie con diferentes parámetros de escorrentía (OchoaRodriguez et al., 2013).

El módulo Sobek-Urban-2D ofrece la posibilidad de implementar un modelo $2 \mathrm{D}$ de la superficie que se puede vincular con el modelo $1 \mathrm{D}$ del sistema de alcantarillado, lo que resulta en un modelo de drenaje doble 1D-2D. Sobek-Urban-2D simula el flujo terrestre utilizando una cuadrícula rectangular (que permite cuadrículas anidadas de menor resolución). Se basa en las ecuaciones completas de Saint Venant. Cuando se implementa un modelo 2D de la superficie en Sobek, es posible aplicar la lluvia al modelo a través de subcuencas o directamente en la superficie. Sin embargo, de manera similar a InfoWorks CS-2D, Sobek-Urban-2D no permite el modelado de lluvia-escorrentía (es decir, estimación de escorrentía) cuando la lluvia se aplica directamente sobre el modelo 2D de la superficie.

Se puede encontrar más información sobre este paquete de software en https://www.deltares.nl/en/software/module/sobek1dflow-urban/.

Canoe es un paquete de software comercial dedicado a la hidrología urbana de uso común en Francia. Es el resultado de un convenio entre dos de los softwares franceses más utilizados en ese país en el campo de la hidrología urbana: CEDRE, desarrollado por el laboratorio URGC-Urban Hydrology de INSA Lyon y CAREDAS, desarrollado por SOGREAH.

Es un paquete de modelado semi-distribuido, similar a InfoWorks CS. Al igual que en InfoWorks CS, las subcuencas pueden comprender diferentes tipos de superficie, cada uno de los cuales tiene diferentes parámetros de escorrentía y tiempos de respuesta. En Canoe, el flujo en el sistema de alcantarillado se modela con la ayuda de una aproximación numérica de la forma más completa de las ecuaciones de Saint-Venant (es decir, enfoque de onda dinámica). Canoe no permite el modelado 2D de la superficie urbana; sin embargo, permite el modelado $1 \mathrm{D}$ de la superficie. Se puede encontrar más detalles sobre este paquete de software en el manual del usuario (Allison et al., 2005, citado en Ochoa-Rodriguez et al., 2013).

A partir de la recopilación presentada anteriormente se puede observar lo siguiente: 
- Existe una gran variedad de modelos hidrológicos aplicados a áreas urbanas principalmente orientados al cálculo de la red de drenaje/conductos pluviales.

- SWMM se presenta como el modelo hidrológicohidráulico disponible gratuitamente más completo para el ámbito urbano. Por el mismo motivo, es utilizado en varios modelos con licencia paga.

- Si se tiene en cuenta modelos totalmente distribuidos disponibles gratuitamente para aplicar al área urbana se podría mencionar actualmente al modelo Itzï, sin embargo, al comenzar con el trabajo actual (año 2014) no estaba disponible dicho proyecto. Por otro lado, el modelo Multihydro que combina el modelo TREX con SWMM aún a la fecha de finalización del trabajo (Finales de 2019) no se encontraba libremente a disposición para su descarga.

Por dichos motivos, se procedió a utilizar de manera combinada el modelo TREX para modelar la transformación lluvia-escurrimiento y el flujo del escurrimiento superficial y el modelo SWMM para modelar la red de drenaje pluvial.

\section{El modelo TREX}

TREX (Two-dimensional Runoff, Erosion, and eXport model) es un modelo bidimensional de escurrimiento, erosión y transporte de sedimentos y contaminantes (England et al., 2007; Velleux et al., 2008). Está basado en el modelo de cuencas CASC2D. Los procesos hidrológicos simulados son: precipitación, intercepción, infiltración y pérdidas por transmisión en el cauce, almacenamiento, flujo superficial y en canales y derretimiento de nieve.

Este modelo utiliza la relación de Green y Ampt para definir la infiltración en la superficie del terreno y en los cauces, y a partir del balance, el flujo superficial. Este flujo está gobernado por las leyes de conservación de la masa y de la cantidad de movimiento. TREX utiliza la aproximación de la onda difusiva unidimensional en cauces $\mathrm{y}$ bidimensional en la superficie de la cuenca, suponiendo que el flujo es turbulento y que la resistencia al flujo se puede describir utilizando la formulación de Manning (Stehli et al., 2012).

Para simular el proceso hidrológico, TREX plantea una solución numérica explícita de las ecuaciones de balance de masa mediante la segmentación de la cuenca en elementos cuadrados iguales, a los cuales se le asignan los parámetros relativos a las características de infiltración del suelo y coeficientes de rugosidad. Este modelo es de código libre, está escrito en el lenguaje de programación C y está disponible en la página web de la Universidad Estatal de Colorado, tiene una fuerte base física en la conceptualización de los procesos superficiales, pero no considera los procesos subsuperficiales y subterráneos (Jorquera et al., 2012).

A continuación, se describen resumidamente los procesos hidrológicos representados por el modelo TREX (Velleux et al., 2008).

El volumen bruto de precipitación $\left(V_{g}\right)$ que llega a la superficie en función del tiempo, es igual a la intensidad de precipitación bruta $\left(i_{g}\right)$ por el área de la región donde ocurre la precipitación $\left(A_{s}\right)$. El volumen neto $\left(V_{n}\right)$ resulta de descontarle al bruto el volumen interceptado $\left(V_{i}\right)$. El balance se explicita en la ecuación (1).

$\frac{\delta V_{g}}{\delta t}=i_{g} \cdot A_{s} \rightarrow V_{n}=V_{g}-V_{i}$

Según el volumen interceptado sea mayor o menor al volumen bruto, habrá o no volumen neto de agua sobre la superficie de la cuenca.

Para definir la infiltración de agua en la superficie de la cuenca como así también en los cauces que la forman, el modelo TREX utiliza la relación de Green y Ampt (1911, citado por Chow et al., 1994).

$f=K_{h} \cdot\left(1+\frac{\Psi\left(1-S_{e}\right) \theta_{e}}{F}\right)$

donde $K_{h}$ es la conductividad hidráulica, $\psi$ es la altura de succión del frente de saturación (altura de succión capilar), $S_{e}$ es el porcentaje de saturación efectiva del suelo, $\theta_{e}$ la porosidad efectiva del suelo, $f$ la tasa de infiltración y $F$ la altura de infiltración acumulada, vinculadas a través de:

$f=\frac{d F(t)}{d t}$

lo cual da lugar a una relación no lineal resuelta a través de un método iterativo (Chow et al., 1994).

La fracción de precipitación no retenida ni infiltrada genera un flujo superficial, tanto mantiforme (bidimensional) como en canales (unidimensional). El flujo superficial puede ocurrir cuando la 
profundidad del agua en el plano terrestre supera el umbral de almacenamiento de la depresión. La ecuación de continuidad para el flujo en dos dimensiones gradualmente variado, sobre un plano rectangular en coordenadas $(\mathrm{x}, \mathrm{y})$ es:

$\frac{\delta h}{d t}+\frac{\delta q_{x}}{d x}+\frac{\delta q_{y}}{d y}=i_{n}-f+W=i_{e}$

donde $h$ es la altura de flujo superficial, $W$ es un término fuente/sumidero (descarga/aporte unitario puntual), $i_{n}$ es la intensidad de precipitación neta, obtenida a partir de (1), $i_{e}$ es la intensidad de precipitación en exceso, y $q_{x}, q_{y}$ son los caudales por unidad de ancho en la dirección x o y. Suponiendo que el flujo es turbulento, la resistencia se puede describir utilizando la formulación de Manning (Chow et al., 1994) tanto para los flujos bidimensionales como unidimensionales. La ecuación (4) es discretizada en el espacio por el método de diferencias finitas, mientras que para la integración temporal se utiliza el método de Euler.

\section{El modelo SWMM}

El programa SWMM (Storm Water Management Model) es un proyecto de software mantenido en la actualidad por la USEPA (U.S. Environmental Protection Agency). Este modelo ha sido especialmente desarrollado para la simulación de sistemas de desagües pluviales y cloacales en forma combinada o separada (EPA, 2005).

Este modelo permite interpretar el comportamiento hidrológico de las cuencas de aportes y la respuesta hidrodinámica del sistema de desagüe. Esta es la principal diferencia respecto de los modelos hidrológicos - hidráulicos estándar, los cuales no consideran las perturbaciones de aguas abajo hacia aguas arriba.

SWMM utiliza para el tránsito de los hidrogramas métodos hidrológicos e hidráulicos, estos últimos consideran las ecuaciones de Saint-Venant en su forma completa. La posibilidad de modelar el tránsito hidráulico resulta fundamental en la simulación de desagües donde las condiciones de aguas abajo influyan sobre el escurrimiento en el sistema, como por ejemplo en tramos de baja pendiente o aguas arriba de conductos de escasa capacidad.

SWMM está compuesto por diferentes módulos (adaptado del manual de referencia de SWMM) (EPA, 2015):
- El Módulo Atmosférico, desde la cual se analiza la lluvia caída y los contaminantes depositados sobre la superficie del suelo, que se analiza en el Módulo de Superficie del Suelo.

- El Módulo de Superficie del Suelo, que se representa a través de uno o más objetos cuenca. Estos objetos reciben la precipitación del Módulo Atmosférico en forma de lluvia o nieve; y generan flujos de salida en forma de infiltración para el Módulo de Aguas Subterráneas y también como escorrentía superficial y cargas de contaminantes para el Módulo de Transporte.

- El Módulo de Aguas Subterráneas recibe la infiltración del Módulo de Superficie del Suelo y transfiere una parte de la misma como flujo de entrada para el Módulo de Transporte.

- El Módulo de Transporte contiene una red con elementos de transporte (canales, tuberías, bombas y elementos de regulación), unidades de almacenamiento y tratamiento que transportan el agua hacia los Nudos de Vertido o salidas del sistema. Los flujos de entrada de este Módulo pueden provenir de la escorrentía superficial, de la interacción con el flujo subterráneo, de los caudales sanitarios correspondientes a periodos sin lluvia, o de hidrogramas de entrada definidos por el usuario. Los componentes del Módulo de Transporte se modelan con los objetos Nudos y Conducciones.

Las conexiones son nudos del sistema de drenaje donde se conectan diferentes líneas entre sí. Físicamente pueden representar la confluencia de canales superficiales naturales, pozos de registro del sistema de drenaje, o elementos de conexión de tuberías. Los aportes externos de caudal entran en el sistema a través de las conexiones. El exceso de agua en un nudo se traduce en un flujo parcialmente presurizado mientras las conducciones conectadas se encuentren en carga. Este exceso de agua puede perderse completamente del sistema o por el contrario estancarse en la parte superior para posteriormente volver a entrar de nuevo en la conexión.

Los conductos son tuberías o canales por los que se desplaza el agua desde un nudo a otro del sistema de transporte. Es posible seleccionar la sección transversal las distintas variedades de geometrías abiertas y cerradas definidas en el programa.

SWMM emplea la ecuación de Manning para establecer la relación entre el caudal que circula por 
el conducto, la sección del mismo, su radio hidráulico y la pendiente tanto para canales abiertos como para conductos cerrados parcialmente llenos.

\section{MODELACIÓN EN CONJUNTO DE TREX CON SWMM}

De la aplicación del modelo TREX en áreas urbanas (Giangola-Murzyn, 2013, Stehli et al., 2016) se verifica que el mismo no incluye las formulaciones para simular el funcionamiento de las bocas de tormenta y los conductos cerrados. Como estos elementos son de gran importancia en la dinámica de las cuencas urbanas, se procedió a la modificación del código fuente de TREX para que simule el funcionamiento de las bocas de tormenta. Estas modificaciones fueron realizadas en el módulo de infiltración. Existirán celdas en donde al haber escurrimiento superficial se infiltra una parte del mismo dependiendo el tipo y característica de boca de tormenta a modelar. De este modo, se simula el caudal insumido en una boca de tormenta.

En las bocas de tormenta a cordón abierto en un punto intermedio se estableció la ecuación de Guo (1997, presentada en Riccardi, 2004) en la cual en primer lugar se calcula la longitud teórica de la boca de tormenta $L_{t}$ en función del caudal total $Q_{0}$ la pendiente longitudinal $S_{l}$, el coeficiente de rugosidad de Manning $n$ de la calle y la pendiente transversal $S_{t}$. Luego el caudal insumido $Q_{i}$ en la boca de tormenta depende de la relación de la longitud teórica de la boca de tormenta y la real $L_{c}$.

$$
\begin{aligned}
L_{t} & =0.817 Q_{0}^{0.42} S_{l}^{0.30}\left(\frac{1}{\eta S_{t}}\right)^{0.5} \\
Q_{i} & =Q_{0}\left[1-\left(\frac{L_{c}}{L_{t}}\right)^{1.8}\right]
\end{aligned}
$$

En las bocas de tormenta a cordón abierto en punto bajo se utilizó la ecuación de Bertoni (1995, presentada en Riccardi, 2004) la cual diferencia entre el funcionamiento como orificio o vertedero según la relación del tirante de agua con respecto a la abertura de la boca. Estas relaciones están en función de la longitud de la boca de tormenta $L_{c}$, el tirante de agua $y_{0}$ y la altura de boca de tormenta $h$. En las ecuaciones (7) y (8) se encuentran estas relaciones.

$Q_{i}=1.656 L_{c} y_{0}^{1.5} \quad\left(y_{0} \leq 1.5 h\right)$
$Q_{i}=0.67 L_{c} h\left[2 g\left(y_{0}-\frac{h}{2}\right)\right]^{\frac{1}{2}}\left(y_{0}>1.5 h\right)$

Para aquellas bocas de tormenta horizontales que se encuentran en puntos bajos se estableció la relación de Neenah (1977, presentada en Riccardi, 2004), la cual relaciona el tirante de agua $y_{0}$, con un coeficiente adimensional $K$ que depende de la pendiente transversal y la pendiente longitudinal. Para el caso, se fijó una pendiente transversal de 2.5 \% de forma tal que el coeficiente $K$ dependerá solamente de la pendiente longitudinal de la calle. La ecuación (9) muestra la relación de Neenah. Se obtuvo una relación para el coeficiente $K$ para una rejilla de un ancho de $0.45 \mathrm{~m}$ y un largo de $0.90 \mathrm{~m}$. Tanto la ecuación (9) como la relación obtenida del $K$ fueron ingresadas al código fuente de TREX.

$Q_{i}=0.205 K y_{0}^{5 / 3}$

Para las bocas de tormenta horizontales situadas en puntos bajos se utilizó la relación de Guo (1997, descripta en Riccardi, 2004). En las relaciones descriptas se establecen nuevas variables; $A_{u}$ es el área útil de la abertura horizontal y $P_{R}$ es el perímetro vertedero de la reja. En las ecuaciones (10) y (11) se encuentran las relaciones dependiendo si la reja trabaja hidráulicamente como vertedero o como orificio.

$Q_{i}=1.656 y_{0}^{1.5} P_{R}\left(y_{0} \leq 1.792\left(A_{u} / P_{R}\right)\right)$
$Q_{i}=2.91 y_{0}^{0.5} A_{u} \quad\left(y_{0}>1.792\left(A_{u} / P_{R}\right)\right)$

Finalmente, también se ingresaron las relaciones para las bocas de tormenta combinadas. Para este tipo de bocas de tormenta, se utilizaron las mismas ecuaciones anteriormente descritas incorporando el procedimiento adecuado para el cálculo de las mismas. En el caso de las bocas que se encuentren en un punto intermedio se calcula en primer lugar el caudal interceptado por la rejilla, con el caudal excedente se calcula la longitud teórica de la boca de cordón abierto, luego la eficiencia de esta boca de tormenta y finalmente el caudal total insumido en la boca de tormenta mixta será la suma del caudal insumido por la rejilla más el caudal insumido por la boca de tormenta abierta. Para el caso de que esté ubicada en punto bajo se calcula el caudal insumido por los dos tipos de elementos (horizontal y vertical) de forma independiente y luego el caudal total es la suma de los dos caudales. 
Para ingresar las relaciones anteriores en TREX se modificaron tres archivos del código fuente del programa, entre ellos, el archivo Infiltration-r5.c donde se encuentran las relaciones del cálculo de infiltración, luego los archivos que manejan la memoria y declaraciones del programa: trex_water_declarations.h y trex_water_definitions.h.

Previamente se intentó realizar las modificaciones en el módulo de escurrimiento superficial de TREX, tratando de usar el modelo con múltiples salidas del sistema dentro de la cuenca. Sin embargo, esta propuesta no tuvo éxito.

Las modificaciones realizadas fueron probadas yverificadas con ejemplos teóricos encontrados en distintos libros especializados en la temática (Mays, 2001).

Posteriormente, se estableció un procedimiento de modelación en conjunto con SWMM para simular los conductos enterrados como así también los arroyos y cauces naturales. En primer lugar se modeló la cuenca con el modelo TREX teniendo en cuenta las bocas de tormenta, es decir, con TREX se modelaron los procesos hidrológicos superficiales. Los cauces a cielo abierto en TREX se representaron como un tipo de suelo especial en el cual se infiltra todo el escurrimiento que llega a esa celda. Luego, con SWMM se modelaron los elementos hidráulicos del sistema, esto es tanto las conducciones cerradas como los canales y arroyos,teniendo como ingreso las salidas de TREXcorrespondientes a las bocas de tormenta y a los arroyos o canales a cielo abierto (Figura 1 ).

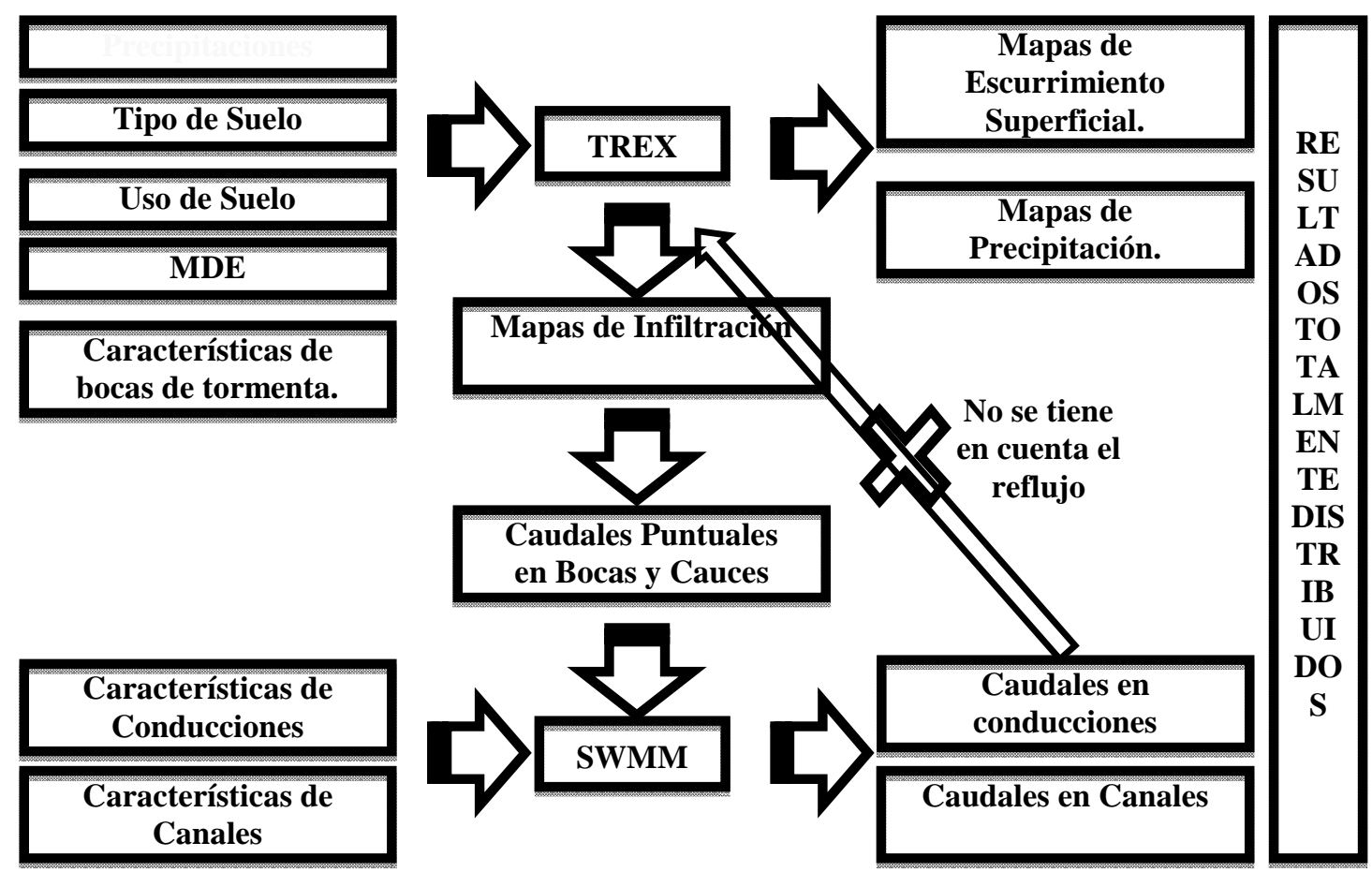

Figura 1. Esquema de procedimiento de modelación distribuida en áreas urbanas con TREX modificado y SWMM.

Al implementar este procedimiento, se desarrollaron programas ad-hoc para extraer de los mapas de infiltración de salida de TREX los valores de caudales a ingresar en cada nodo (conexión) de boca de tormenta o cauce de SWMM.

\section{CASO DE APLICACIÓN}

El caso de estudio presentado en este trabajo es la Cuenca del Rio Cascavel, en el municipio de Guarapuava localizada en el Centro Sur del Estadode Paraná, Brasil. La cuenca tiene un área aproximada de $81 \mathrm{~km}^{2}$. De su área total, $55 \mathrm{~km}^{2}$ es decir, 69 \% están dentro del perímetro urbano oficial de la localidad de Guarapuava (Figura 2).

Para la modelación de la cuenca se utilizaron los siguientes datos de entrada, los cuales fueron procesados para realizar los modelos en TREX y SWMM. 


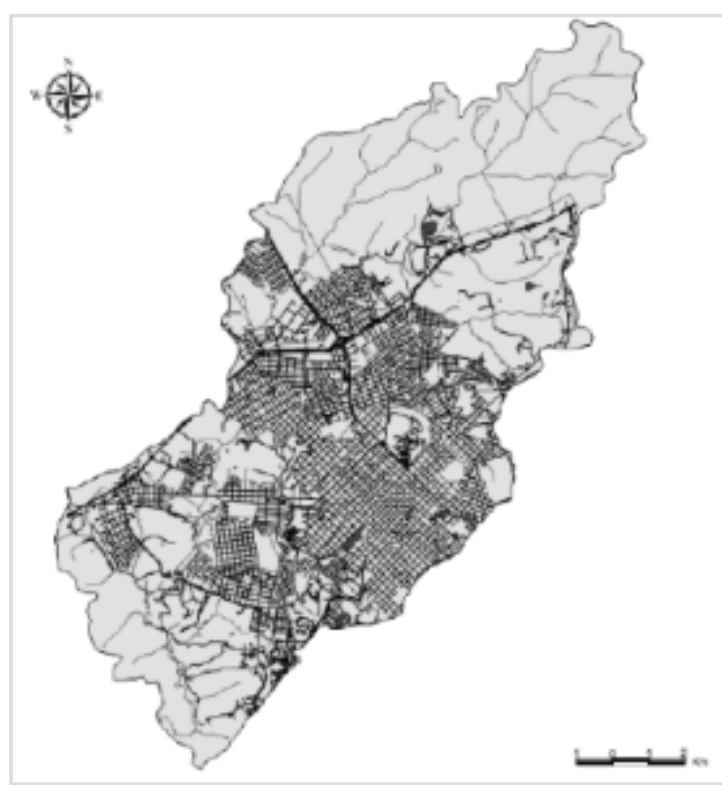

Figura 2. Cuenca del Río Cascavel - Adaptado de Gomes (2014).

Topografía: El modelo digital de elevación fue elaborado a través de la interpolación lineal de datos relevados por distintas campañas efectuadas por la Municipalidad de Guarapuava, asignadas por la Universidad Estatal de Centro Oeste (UNICENTRO) de Brasil.

Uso y tipo de suelo: Se utilizaron los mapas elaborados por Gomes (2014). En lo que respecta al mapa de uso de suelo, el mismo es resultado del procesamiento de imágenes Rapideye del 27/09/2011. De la clasificación se evidencia que predominan las clases de vegetación rastrera/cultivo y las áreas construidas con un porcentaje de $26.7 \%$ y 35.8 \% respectivamente, del área total. Los tipos de suelo en la cuenca son tres: lattosolos brunos y nittosolos brunos y litólicos.

Precipitaciones: Se seleccionaron eventos históricos correspondientes a una estación pluviográfica y dos estaciones pluviométricas. Las fechas de los eventos seleccionados van desde Junio de 2011 hasta diciembre de 2013, en correspondencia a la existencia de datos de caudales líquidos.

Caudales líquidos: Se utilizaron para la calibración los resultados de medición de un limnígrafo automático que se encuentra en coincidencia a la estación pluviográfica, dentro de la cuenca Cascavel (no en el punto de cierre de la misma).Los valores medidos del caudal líquido en la estación Cascavel se encuentran con un intervalo de 15 minutos.
En el mapa de tipo de suelo se agregaron los tipos correspondientes a las distintas bocas de tormenta existentes en la cuenca, las cuales fueron relevadas a través de Google Street View ${ }^{\circledR}$, esto debido a la inexistencia de documentos que indiquen la cantidad y ubicación de las mismas (Figura 3). También se agregaron dos tipos de suelo adicionales, uno para simular un suelo impermeable en correspondencia a las construcciones $\mathrm{y}$ calles pavimentadas $\mathrm{o}$ asfaltadas y otro tipo de suelo totalmente permeable para simular las celdas correspondientes a los arroyos y cauces naturales.

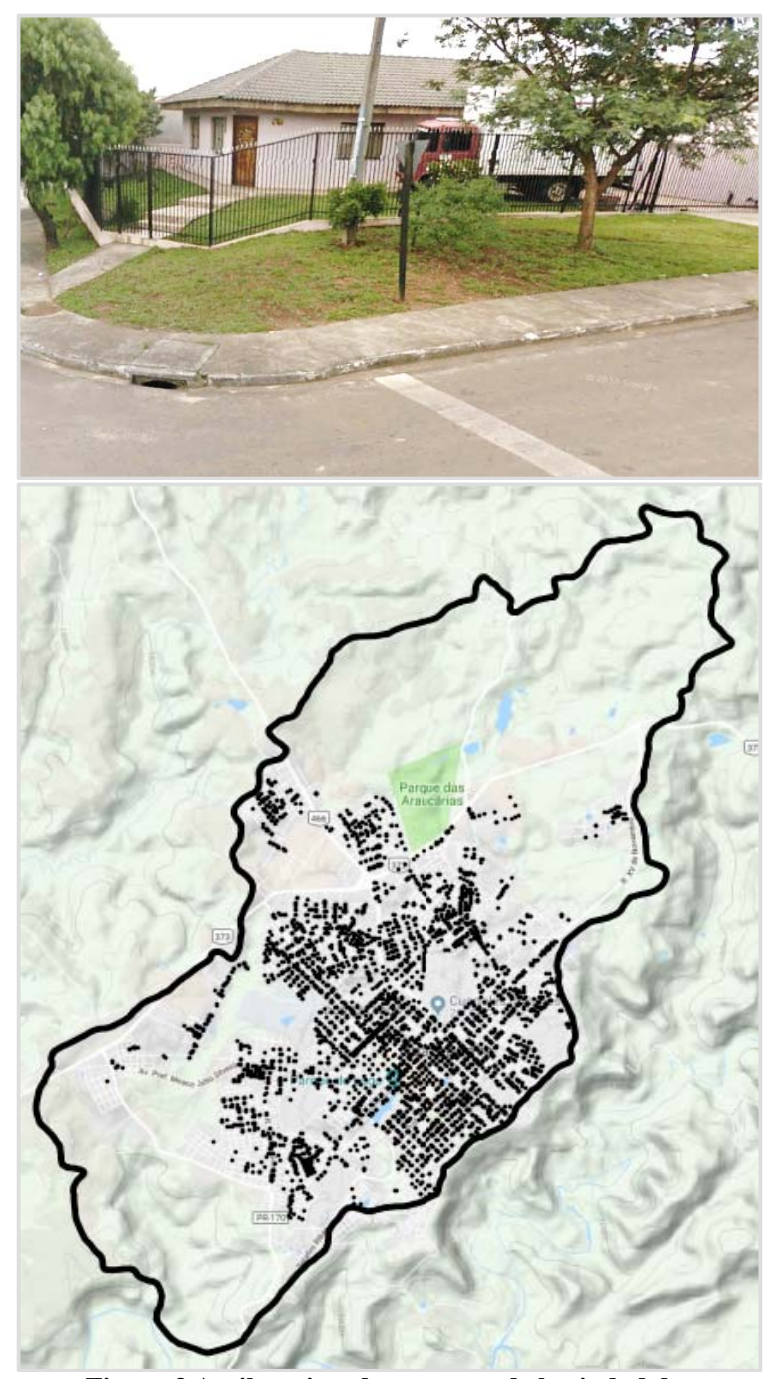

Figura 3.Arriba: vista de una zona de la ciudad de Guarapuava en Street View. Abajo: identificación de lasbocas de tormenta.

Con estos mapas, y el de uso de suelo, se elaboraron los archivos de ingreso para el modelo TREX (Figura 4). Debido a la densidad de bocas de 
tormenta, la resolución base del mapa de uso de suelo (5 m x $5 \mathrm{~m}$ ) y la densidad de levantamientos topográficos dentro de la zona urbana, se eligió realizar las modelaciones con celdas de $5 \mathrm{~m}$ x $5 \mathrm{~m}$ en las áreas urbanas y celdas de $30 \mathrm{~m}$ x $30 \mathrm{~m}$ en áreasrurales, dividiendo la cuenca en 8 subcuencas
(Figura 5). Esto facilitó también el manejo de los datos, especialmente en el modelo TREX, donde se genera gran información de salida (mapas de infiltración, precipitación y escurrimiento superficial a distintos intervalos de tiempo).
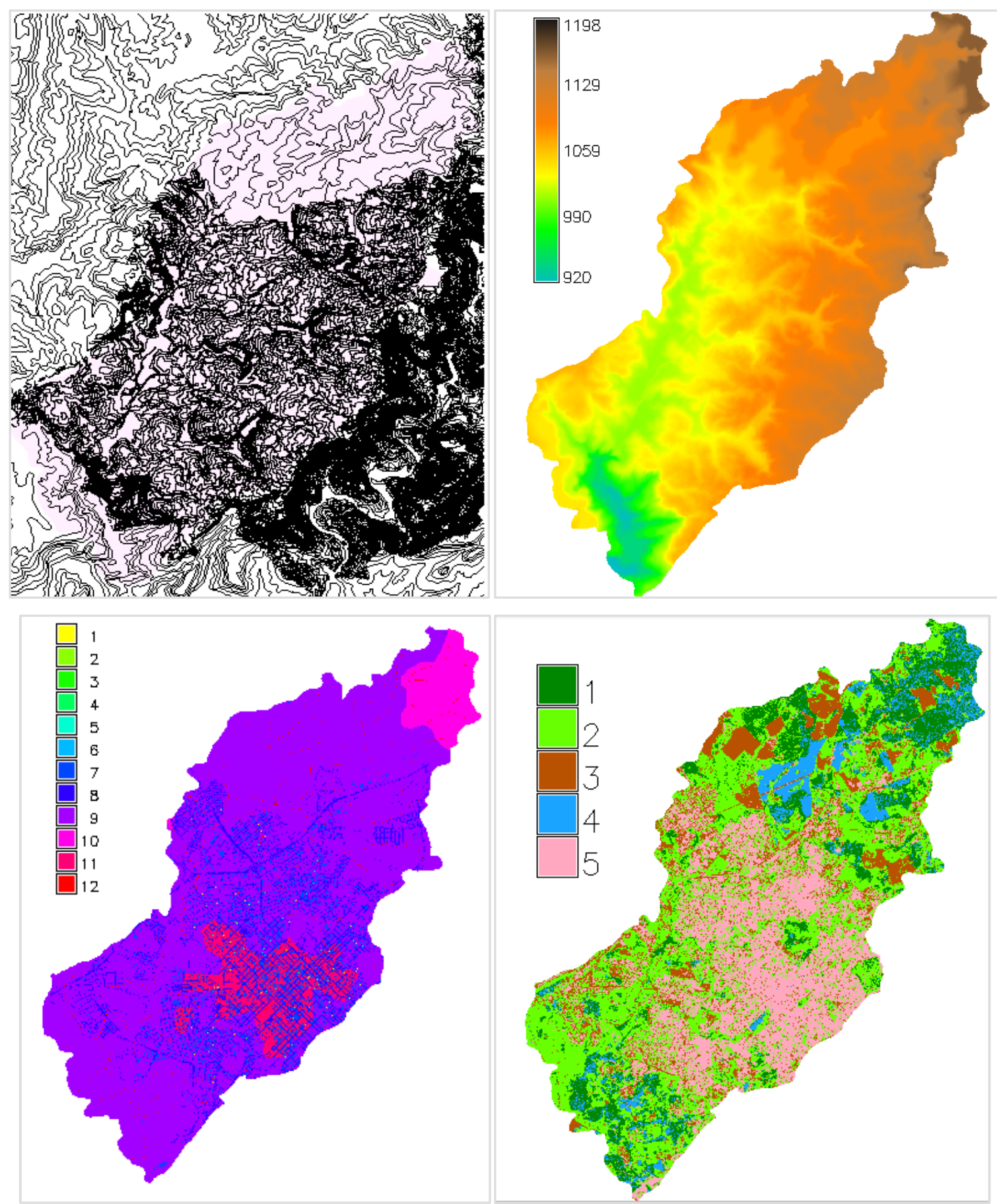

Figura 4. Mapas de ingreso para la modelación de la cuenca de Cascavel en TREX. De izquierda a derecha: curvas de nivel de la cuenca utilizado para producir MDE, MDE, mapa de tipo de suelo y mapa de uso de suelo. 


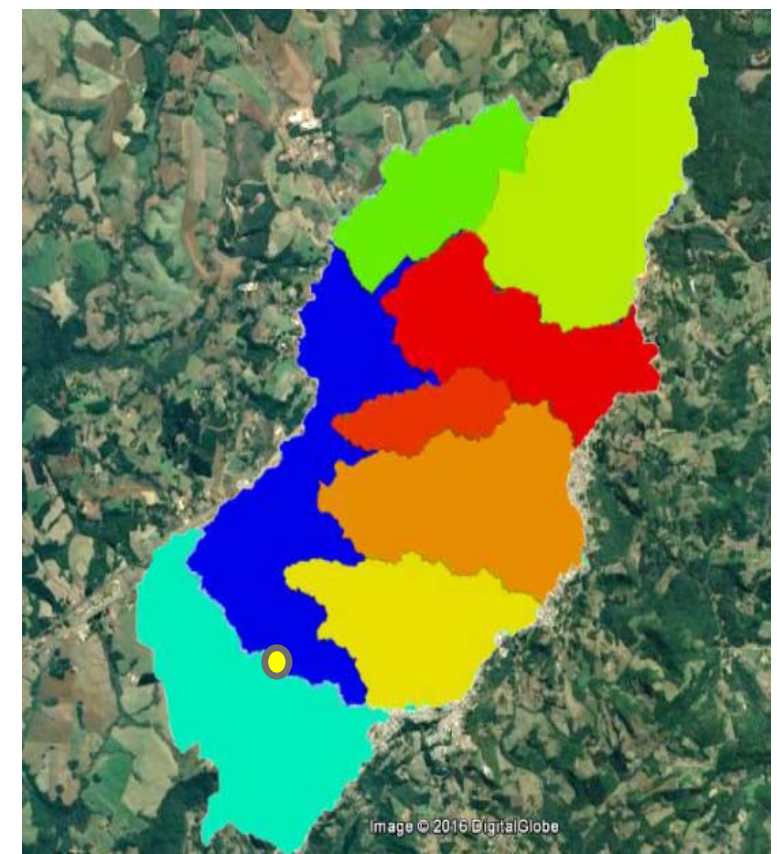

Figura 5. División de la Cuenca del Rio Cascavel en ocho subcuencas para mejorar la trabajabilidad en el modelo TREX.

En SWMM se creó la red de drenaje compuesta por nodos correspondientes a las bocas de tormenta (que tienen como ingreso el flujo infiltrado en TREX en ese tipo de suelo) y nodos cauces (que tienen como ingreso el flujo infiltrado en TREX en el tipo de suelo cauce) y los conductos que representan los cauces. Estos elementos se modelaron utilizando el relevamiento realizado anteriormente para ubicar las bocas de tormenta y por mapas desarrollados por el Instituto Militar donde representan la red de drenaje natural. Al no existir documentación de ubicación y características de conductos, se simuló la red como una red cerrada donde los conductos corren en la parte central de las calzadas y se unen entre ellos en un nodo auxiliar al intersectarse. Las bocas de tormenta descargan a la red a través de un conducto que une a las mismas al nodo auxiliar más cercano. Se procedió de la misma forma para las conexiones conductoscauce (Figura 6).

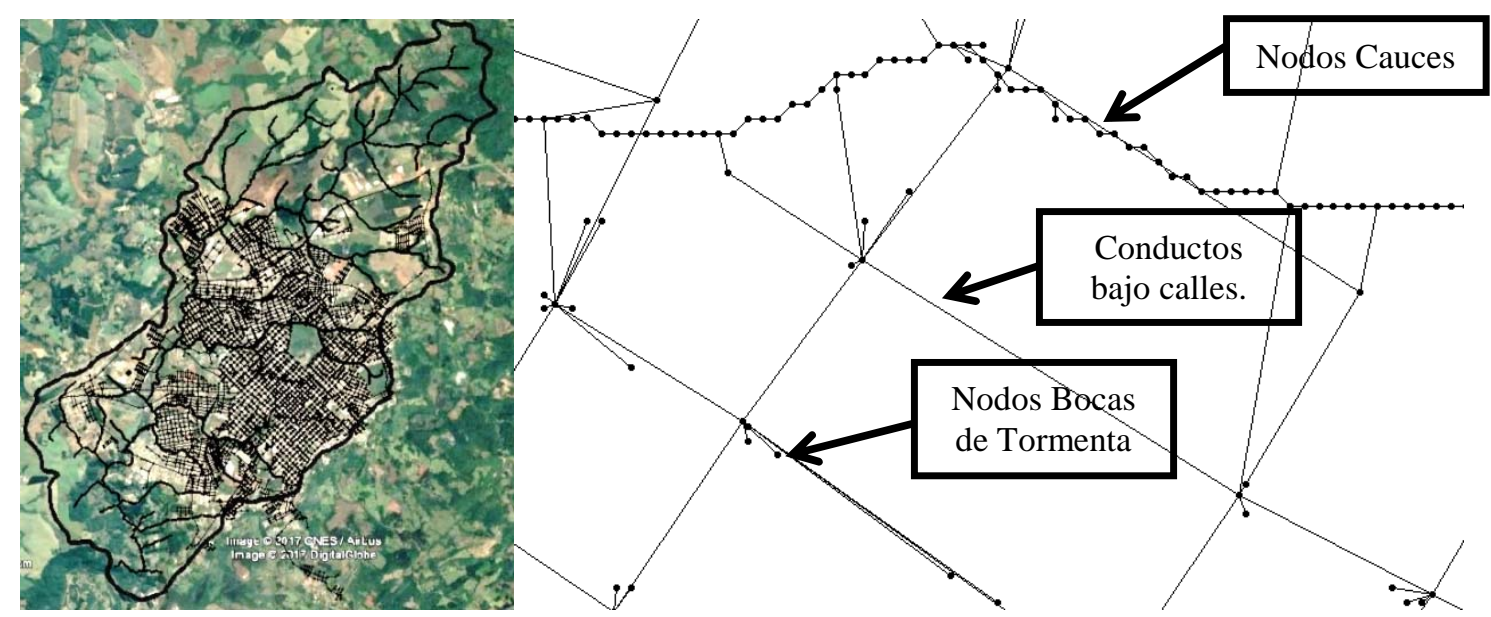

Figura 6. Izquierda vista del modelo completo de SWMM de la Cuenca de Cascavel. Derecha detalle de un sector del modelo.

De esta forma se creó un modelo que contiene 3494 nodos de conexión de conductos de calle (red cerrada), 5285 conductos que simulan las cañerías bajo calles, 4211 nodos que representan las bocas de 
tormenta en la Ciudad de Guarapuava, con su correspondientes conductos de conexión con la red de conductos de drenaje, 15095 nodos correspondientes a celdas cauces con sus correspondientes conductos de conexión, 1 embalse y 15306 datos de ingreso de caudal puntual al sistema. A estos elementos, se les asociaron los parámetros característicos para su adecuada modelación, por ejemplo, en los nodos la cota de fondo (según MDE) y profundidad y en los conductos longitud (según coordenadas de nodos), forma (según relevamiento) y rugosidad (Stehli et al., 2021).

La red de drenaje de conductos cerrados fue simulada como una malla cerrada, esto fue debido al desconocimiento de la traza real de las conducciones. Por este motivo, el método de cálculo hidráulico utilizado en SWMM fue el de la onda dinámica, para poder simular los distintos flujos que se producirán en las conducciones de la malla.

\section{RESULTADOS}

Se presentan a continuación los resultados de la calibración de los eventos seleccionados.

En base a los caudales medidos de cada evento, se extrajo de cada uno el caudal base para poder comparar entre escurrimiento directo medido y escurrimiento directo simulado. Esto es consecuencia, de que en TREX no es posible simular los fenómenos que producen variaciones en los caudales base. Para la separación del escurrimiento directo se utilizó el método de Barnes (Orsolini et al., 2000).
A continuación se presentan los resultados gráficos de los eventos calibrados y los parámetros estadísticos de ajuste (Figura 7 a 12y Tabla 1). En estas figuras los valores medidos han sido registrados por puntos, y el resultado de la simulación con TREX y SWMM con línea continua. Además se agrega el hietograma medio de la cuenca. En Tabla 1, también se presentan los valores de precipitación media caída en toda la cuenca para cada uno de los eventos.

La calibración se realizó de forma manual, ajustando los parámetros de infiltración, rugosidad del flujo mantiforme e intercepción vegetal en TREX (donde se modela la transformación lluvia-caudal) y los parámetros de rugosidad de cauces y conductos en SWMM. En el modelo de infiltración, se debe calibrar el parámetro de déficit de humedad. Este depende de cada evento debido a las condiciones antecedentes de humedad, las cuales se estimaron previamente según los valores de precipitación de los días anteriores al evento simulado y luego se ajustaron para cada evento de forma tal de mejorar los indicadores de evaluación de bondad de ajuste (Tabla 1). En algunos eventos simulados, como el evento 5 y 9 en los días previos existe un valor bajo de precipitación en la cuenca, que no se correspondería con los valores de déficit de humedad calibrado. Sin embargo, se observa por ejemplo en el evento 9 que si se tienen en cuenta 15 días antecedentes al evento, existe un evento extraordinario; del 29/06/2011 al 03/07/2011 se registra en la estación pluviográfica de Cascavel $338 \mathrm{~mm}$ precipitados. En cuanto a la intercepción vegetal, la misma está relacionada en TREX para cada uso de suelo, existiendo una diferencia entre los valores en áreas impermeables/construidas que los sectores boscosos y de praderas.

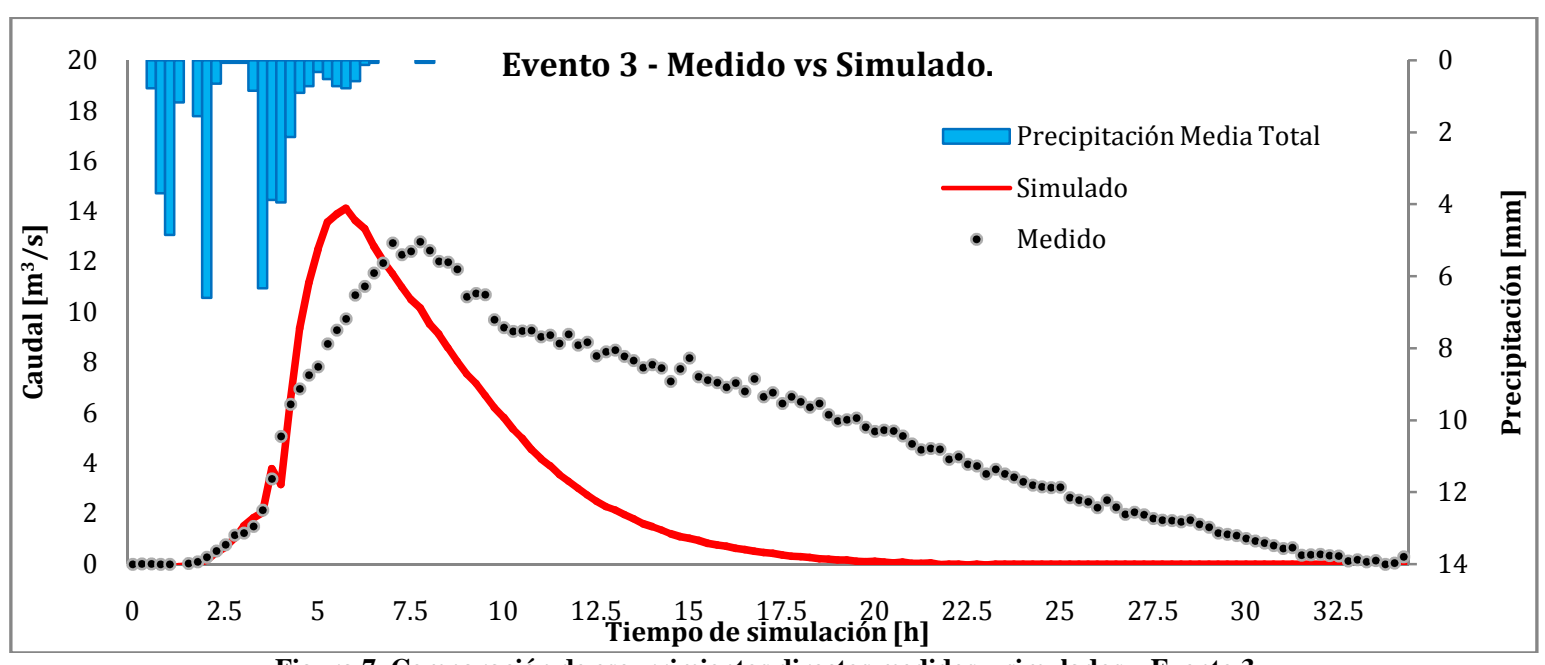

Figura 7. Comparación de escurrimientos directos medidos y simulados - Evento 3. 


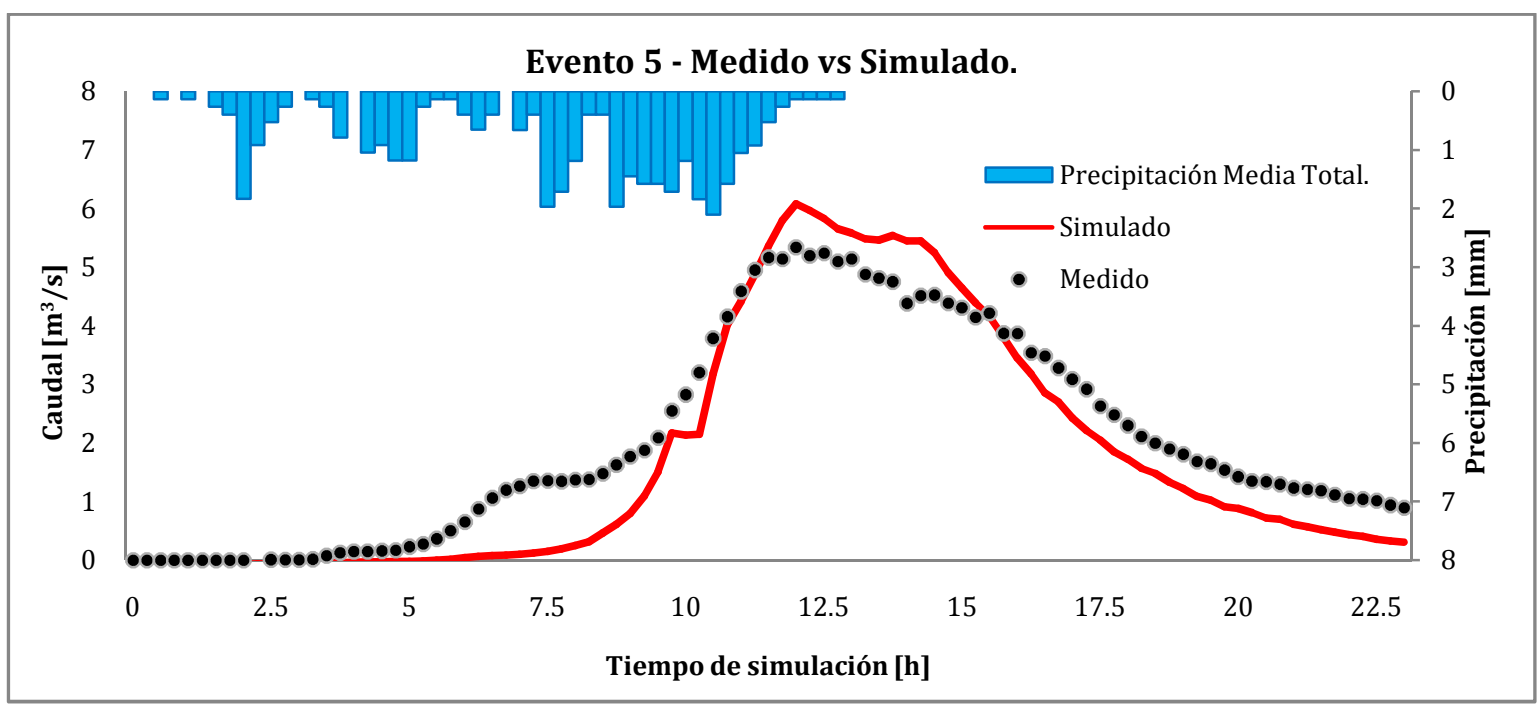

Figura 8. Comparación de escurrimientos directos medidos y simulados - Evento 5.

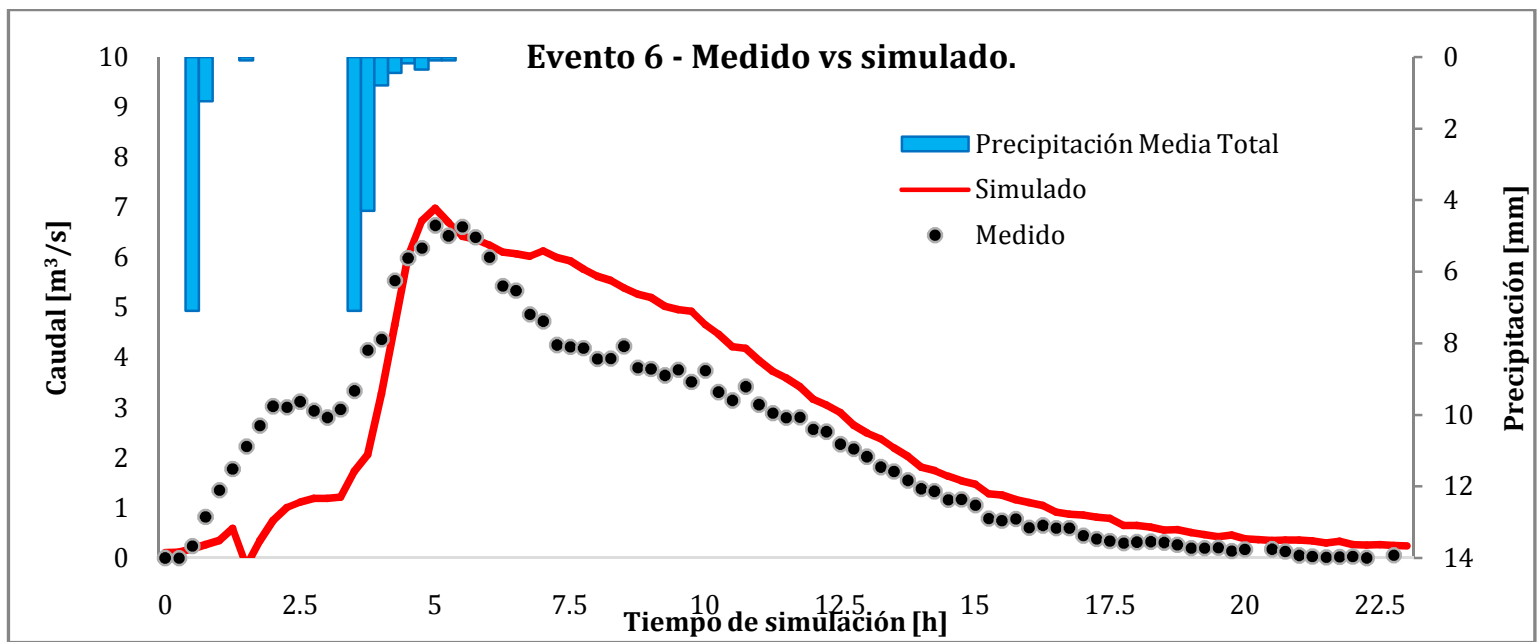

Figura 9. Comparación de escurrimientos directos medidos y simulados - Evento 6.

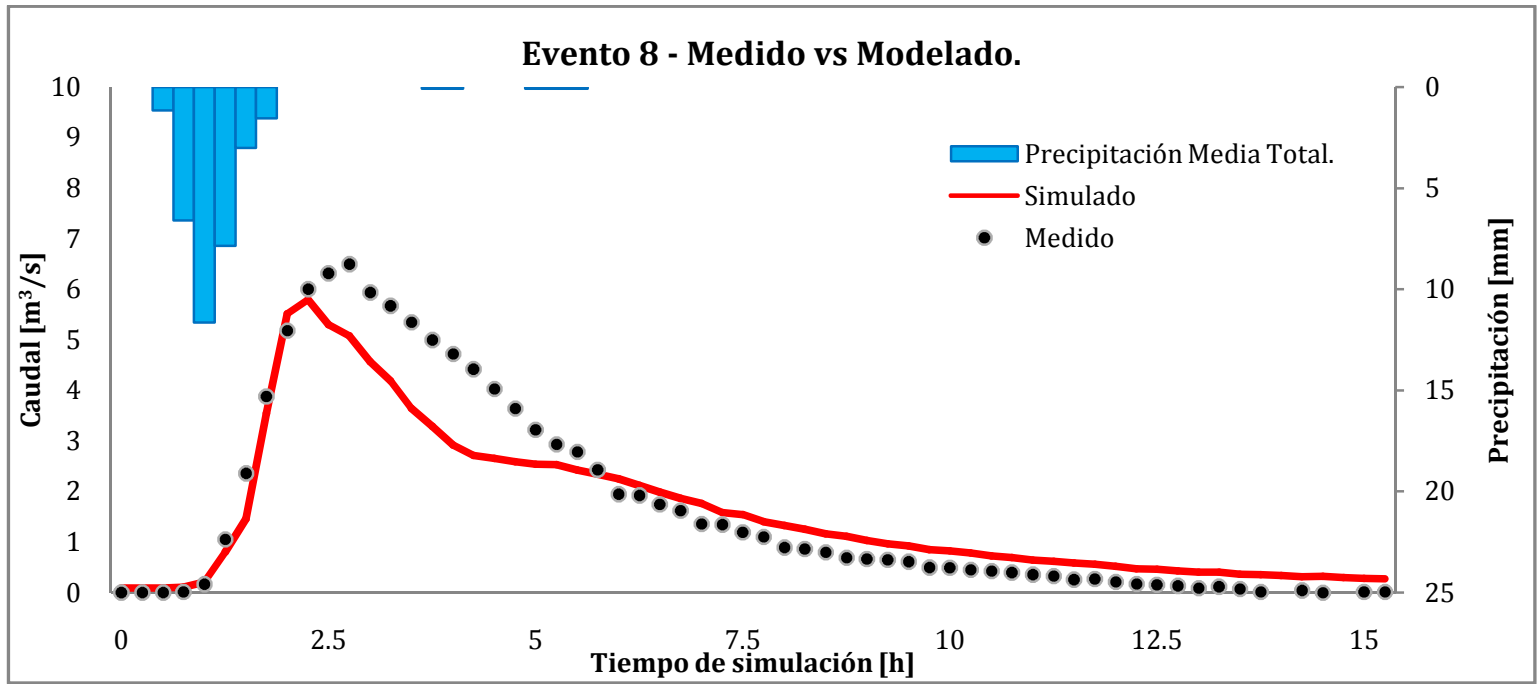

Figura 10. Comparación de escurrimientos directos medidos y simulados - Evento 8 


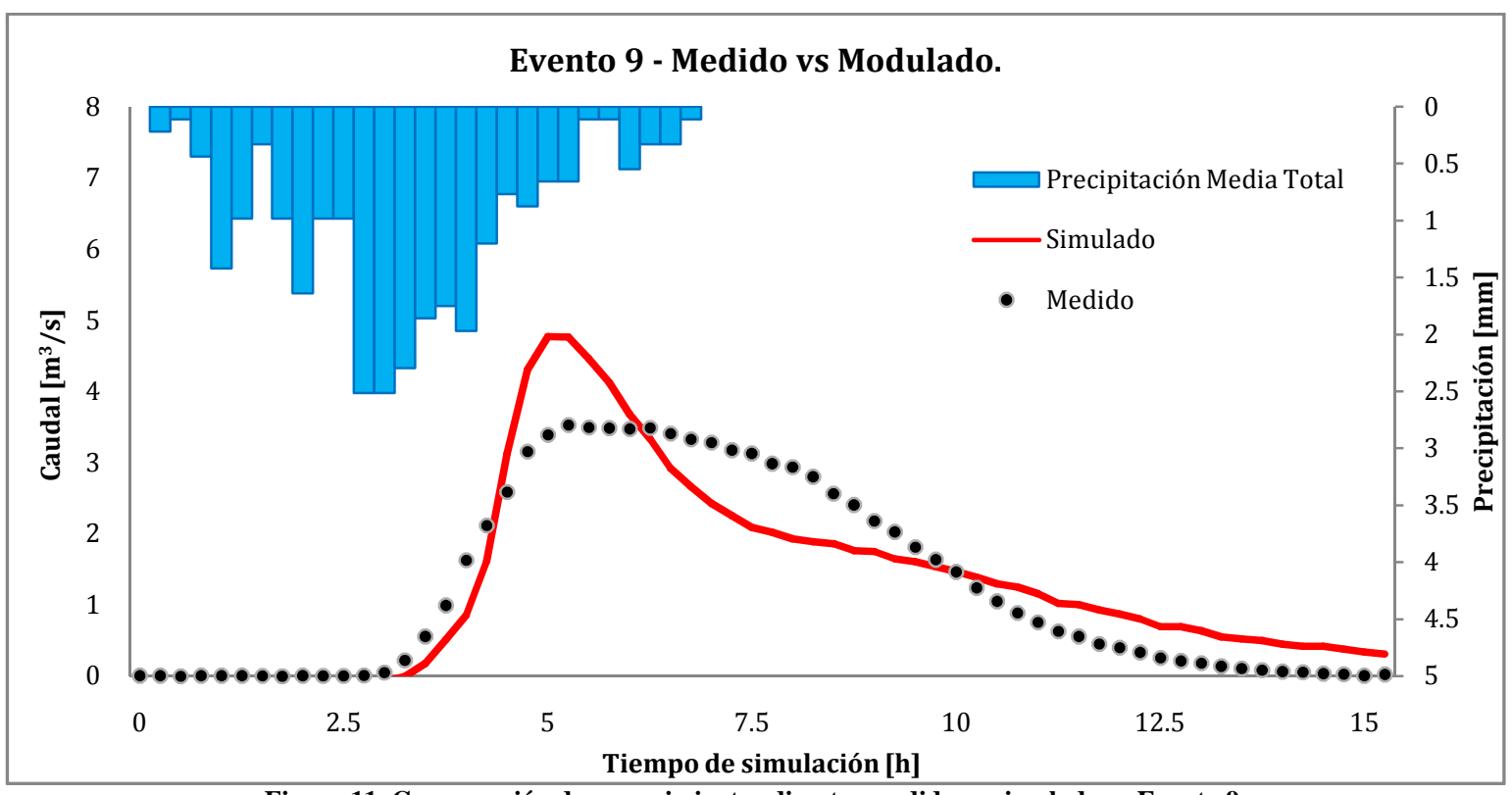

Figura 11. Comparación de escurrimientos directos medidos y simulados - Evento 9.
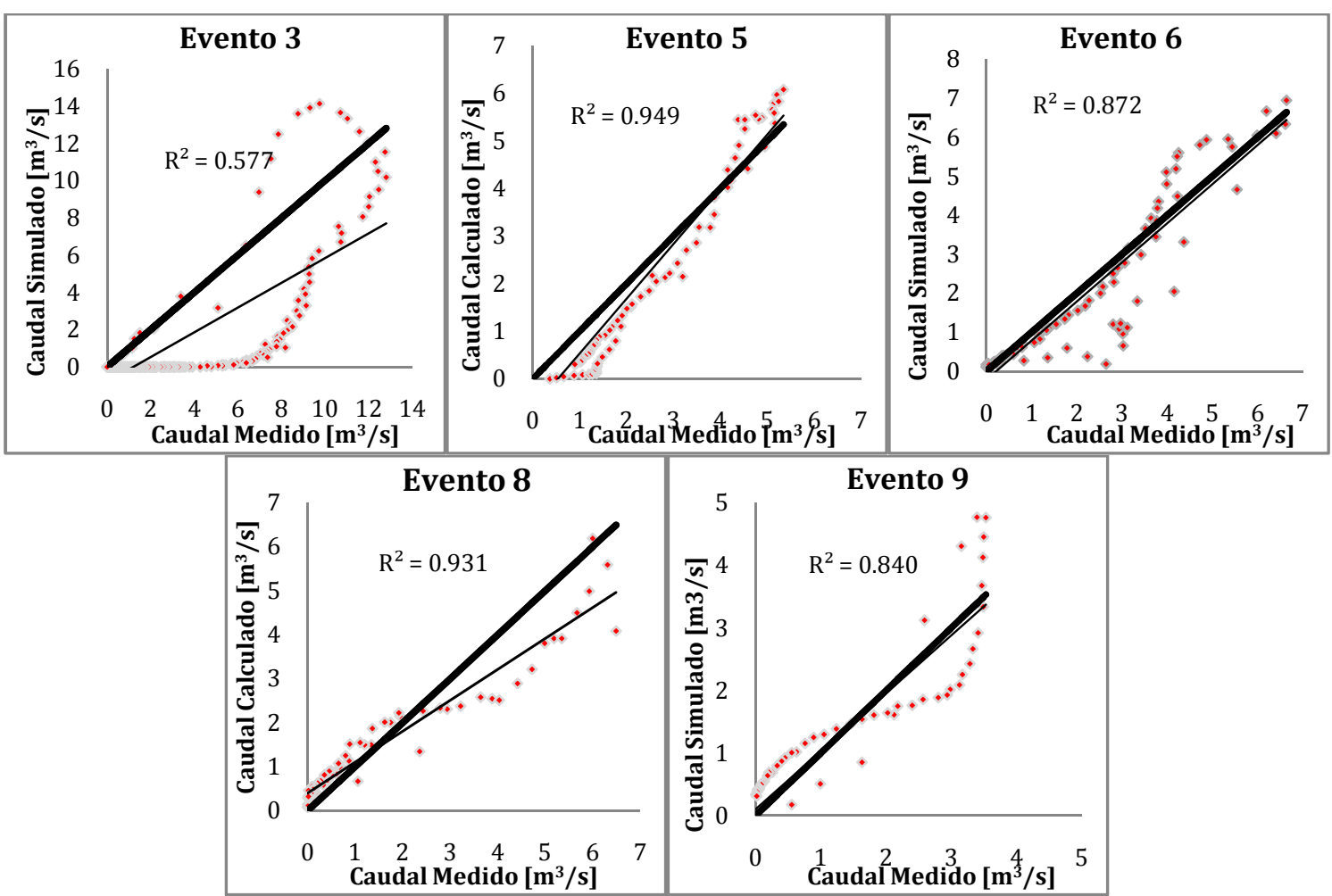

Figura 12 - Gráficos de correlación de cada uno de los eventos calibrados En gris, la línea que representa un ajuste perfecto. 
Tabla 1 - Indicadores estadísticos para evaluación de bondad del ajuste de hidrogramas obtenidos en eventos de calibración.

\begin{tabular}{|c|c|c|c|c|c|c|c|c|c|}
\hline Evento & Fecha & $\begin{array}{c}\mathbf{P}_{\text {media }} \\
\text { evento } \\
{[\mathbf{m m}]}\end{array}$ & $\begin{array}{c}\mathbf{P}_{\text {media }} \\
\mathbf{5} \text { días } \\
\text { previos } \\
\text { [mm] }\end{array}$ & $\mathbf{R}^{2}$ & NSE & $\begin{array}{c}\text { PBIAS } \\
{[\%]}\end{array}$ & Media & $\begin{array}{c}\text { Desviación } \\
\text { estándar }\end{array}$ & $\begin{array}{c}\text { Déficit de } \\
\text { Humedad } \\
\text { calibrado }\end{array}$ \\
\hline Evento 3 & $08 / 01 / 2013$ & 47.86 & 54.57 & 0.545 & -0.649 & 48.26 & 3.22 & 1.796 & 0.050 \\
\hline Evento 5 & $26 / 08 / 2013$ & 36.85 & - & 0.949 & 0.874 & 0.19 & 0.516 & 0.719 & 0.035 \\
\hline Evento 6 & $06 / 01 / 2013$ & 43.61 & 22.35 & 0.872 & 0.842 & 9.19 & 0.207 & 0.455 & 0.070 \\
\hline Evento 8 & $12 / 12 / 2012$ & 32.40 & 2.10 & 0.945 & 0.905 & 4.49 & 0.072 & 0.268 & 0.300 \\
\hline Evento 9 & $17 / 07 / 2011$ & 26.68 & 1.50 & 0.840 & 0.832 & -0.83 & 0.010 & 0.102 & 0.090 \\
\hline
\end{tabular}

En la calibración, se observa en general una aceptable correlación entre los caudales simulados con los observados. Dicha correlación se aprecia observando también los resultados del coeficiente $\mathrm{R}^{2}$. Sin embargo, se observa en el Evento 3 que los valores de NSE y PBIAS se alejan sensiblemente del valor ideal.

Al observar la gráfica de los hidrogramas simulados de los eventos mencionados se puede inferir algunas de las posibles causas de los desvíos entre los valores simulados y los medidos, por ejemplo se aprecia en el Evento 3 la diferencia entre el volumen de escurrimiento simulado con respecto al volumen de escurrimiento medido.En cuanto al evento 6 (Figura 9), no es posible captar un pico inicial de crecida que se da anteriormente al pico principal.

Estos errores pueden deberse a diversos motivos. En el caso de estudio puede atribuirse a la incertidumbre de la distribución temporal de las precipitaciones, ya que, como se mencionó anteriormente, se la adoptó proporcional a la distribución temporal dada por el pluviógrafo. Esto, dependiendo la naturaleza del evento considerado puede tener un cierto grado de error, más todavía en cuencas de un tamaño considerable. Lo anterior, se puede inferir de trabajos anteriores (Weber et al., 2012) en donde se demuestra que el movimiento de la tormenta al ocurrir un evento influye en la salida del sistema hidrológico.

Se analizó también la diferencia porcentual entre el volumen escurrido calculado y el volumen escurrido medido, como así también los caudales picos medidos y simulados. En las Tabla 2 se presenta lo anterior y se puede observar lo que se mencionó anteriormente respecto al evento 3 que no se pudo simular adecuadamente la rama descendente del hidrograma, por lo tanto el volumen escurrido simulado es considerablemente menor al volumen escurrido medido. Teniendo en cuenta también para dicho evento que el caudal pico simulado es levemente superior al medido, se podría inferir que hay algún error en las precipitaciones o en las distribuciones temporal y espacial de las mismas. Adicionalmente, en la Tabla 3 se agregaron el tiempo de ocurrencia de los caudales picos de los hidrogramas medidos y simulados (tiempo desde el inicio de la precipitación de cada evento). En dicha tabla se puede observar que en general los caudales picos de los hidrogramas simulados ocurren en coincidencia o casi en coincidencia con los caudales picos medidos, exceptuando el evento 3 en donde en la simulación el caudal pico se adelanta 2.2 horas con respecto al caudal medido. Esta diferencia en el tiempo del caudal pico sumada a la diferencia del volumen escurrido entre lo simulado y medido en el evento 3, nos permite inferir que puede que exista una diferencia en la precipitación caída, principalmente en su distribución temporal. Esto último teniendo en cuenta que se tiene información de la distribución temporal de las lluvias de una sola de las tres estaciones, en la que existe el pluviógrafo, y luego para las dos estaciones pluviométricas se adoptó dicha distribución temporal.Sin embargo, se puede considerar como aceptable el grado de ajuste obtenido en los eventos seleccionados y para los pares de datos utilizados para la calibración, más aun teniendo en cuenta los grados de incertidumbre, por ejemplo en las precipitaciones y en tipología y características de la red de drenaje subterránea.

Como se mencionó anteriormente, con el procedimiento establecido se pueden obtener resultados distribuidos en toda la cuenca, tanto deescurrimientos superficiales, precipitación e infiltración (TREX) como de flujos en conductos y cauces (SWMM).

Se presentan la visualización de algunos resultados en la extensión de la cuenca (Figuras 13, 14 y 15) y 
la serie de valores calibrados para los eventos seleccionados (Tabla 4).

Se observa también que en general en todos los eventos para los mayores caudales medidos se corresponde caudales simulados aún más grandes que los medidos (se observan por encima de la línea identidad del gráfico, Figura 12) y luego para caudales menores, los caudales simulados son menores que los medidos (puntos por debajo de la línea identidad del gráfico). Esto se observa en todos los eventos en mayor o menor medido, exceptuando el evento 8.Por lo tanto explicaría que para poder lograr un buen ajuste de los hidrogramas lo que se está haciendo es sobredimensionar levemente el pico de crecida y que luego las ramas de ascenso y descenso del hidrograma simulado están por debajo del hidrograma medido. Es decir, que la crecida simulada llega antes al punto de medición que la real y a la vez se evacúa también más rápidamente.Aunque esto último pudiera indicar que se estaría utilizando en la calibración una rugosidad menor a la correcta para que la respuesta de la cuenca sea más lenta, se observa en el proceso de calibración que al utilizar una rugosidad mayor (ya sea de flujo mantiforme, de cauces o de conductos) el pico de crecida se demoraba en demasía en comparación al pico de crecida medido.

Tabla 2. Análisis de diferencias entre volumen escurridos y caudales picos medidos y simulados para los eventos de calibración.

\begin{tabular}{|c|c|c|c|c|c|c|}
\hline & \multicolumn{3}{|c|}{ Volumen Escurrido } & \multicolumn{3}{c|}{ Caudal Pico } \\
\hline & $\begin{array}{c}\text { Medido } \\
{\left[\mathrm{m}^{3}\right]}\end{array}$ & $\begin{array}{c}\text { Simulado } \\
{\left[\mathrm{m}^{3}\right]}\end{array}$ & $\begin{array}{c}\text { Diferencia } \\
{[\%]}\end{array}$ & $\begin{array}{c}\text { Medido } \\
{\left[\mathrm{m}^{3} / \mathrm{s}\right]}\end{array}$ & $\begin{array}{c}\text { Simulado } \\
{\left[\mathrm{m}^{3} / \mathrm{s}\right]}\end{array}$ & $\begin{array}{c}\text { Diferencia } \\
{[\%]}\end{array}$ \\
\hline Evento 3 & 559457.65 & 289472.41 & $-48 \%$ & 12.8 & 13.89 & $9 \%$ \\
\hline Evento 5 & 170679.97 & 144601.87 & $-15 \%$ & 5.34 & 6.081 & $14 \%$ \\
\hline Evento 6 & 188460.82 & 171143.33 & $-9 \%$ & 6.4 & 6.95 & $9 \%$ \\
\hline Evento 8 & 93275.14 & 89543.03 & $-4 \%$ & 6.49 & 5.63 & $-13 \%$ \\
\hline Evento 9 & 70888.50 & 71474.18 & $1 \%$ & 3.53 & 4.77 & $35 \%$ \\
\hline
\end{tabular}

Tabla 3. Tiempo de ocurrencia de caudal pico de crecida en hidrograma medido y simulado para los eventos de calibración.

\begin{tabular}{|c|c|c|c|}
\hline & \multicolumn{3}{|c|}{ Tiempo de ocurrencia de pico. } \\
\hline Evento 3 & $\begin{array}{c}\text { Medido } \\
\text { [horas] }\end{array}$ & $\begin{array}{c}\text { Simulado } \\
\text { [horas] }\end{array}$ & $\begin{array}{c}\text { Diferencia } \\
\text { [horas] }\end{array}$ \\
\hline Evento 5 & 7.70 & 5.50 & -2.20 \\
\hline Evento 6 & 12.00 & 12.00 & 0.00 \\
\hline Evento 8 & 5.00 & 5.00 & 0.00 \\
\hline Evento 9 & 5.75 & 2.50 & -0.25 \\
\hline
\end{tabular}

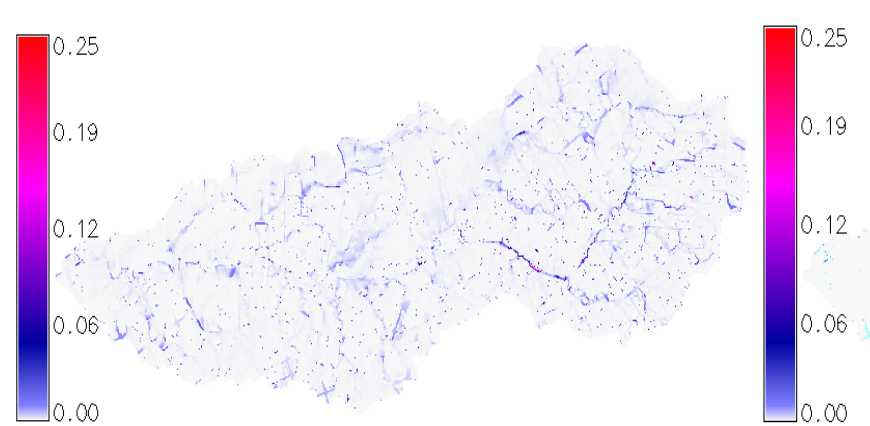

Figura 13. Altura de escurrimientos superficiales [m], subcuenca Barro Preto para los tiempos 10 y 15 horas (imagen izquierda y derecha respectivamente) de iniciada la precipitación - Evento 5. 


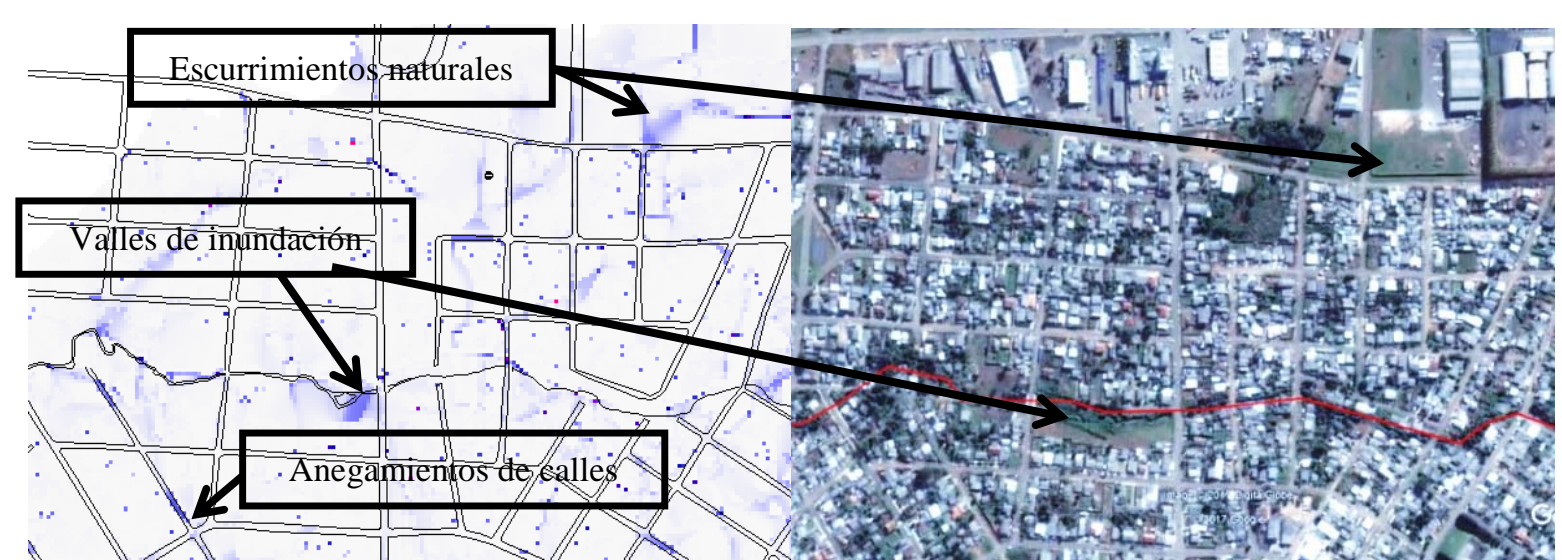

Figura 14. Altura de escurrimientos superficiales, sector de la subcuenca Barro Preto para 10 horas de iniciada la precipitación, comparación con imagen satelital - Evento 5.

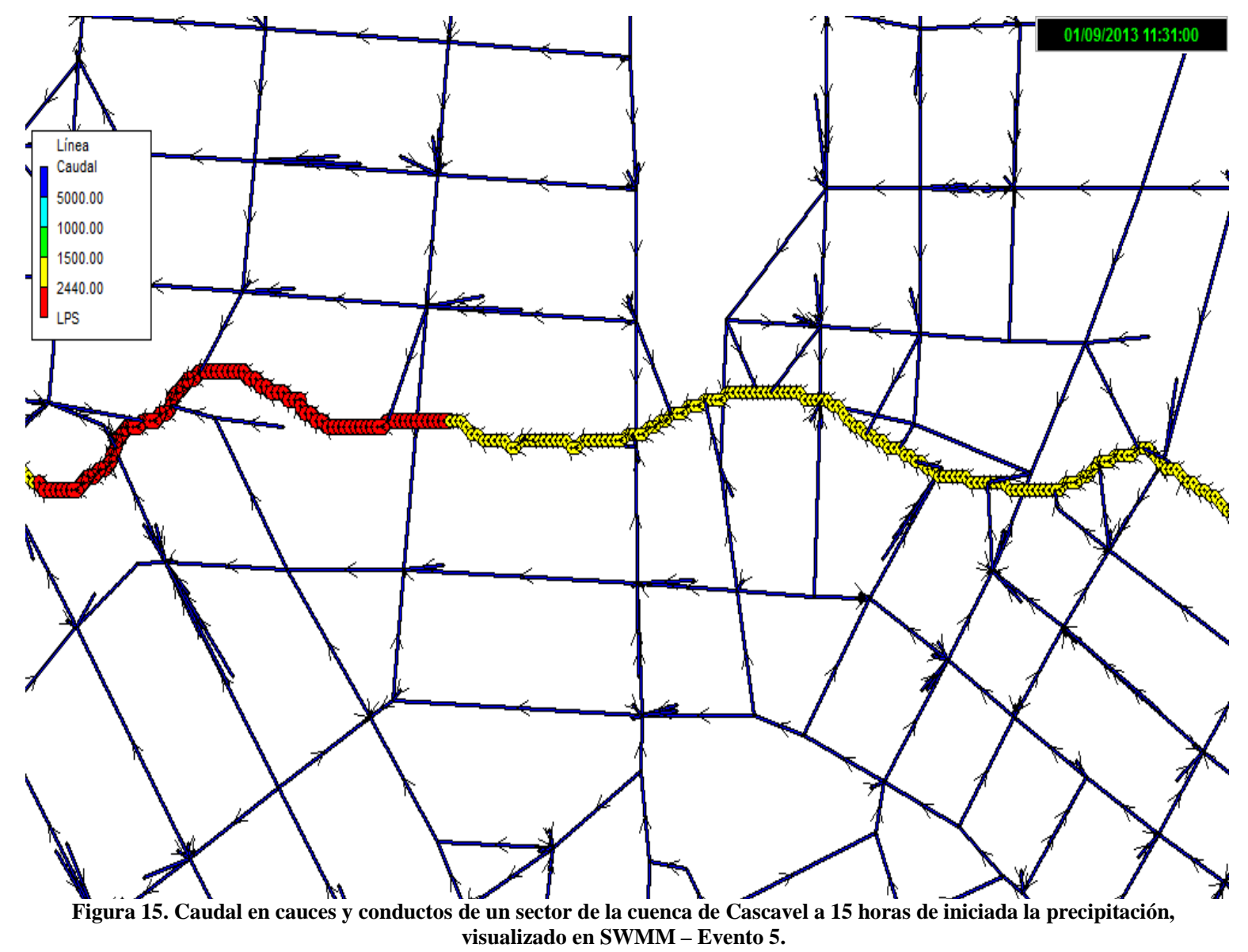


Tabla 4.Valores calibrados de los parámetros de infiltración y rugosidad del modelo TREX.

\begin{tabular}{|c|c|c|c|}
\hline \multicolumn{5}{|c|}{ Parámetros de Infiltración } \\
\hline Tipo de suelos considerados & $\begin{array}{c}\mathbf{K}_{\mathbf{h}} \\
{[\mathbf{m} / \mathbf{s}]}\end{array}$ & $\begin{array}{c}\text { Altura de succión } \\
{[\mathbf{m}]}\end{array}$ & $\begin{array}{c}\text { Porosidad efectiva } \\
\text { [adimensional }\end{array}$ \\
\hline Construcciones (Impermeable) & $12.00 \mathrm{E}-25$ & 0.1903 & 0.010 \\
\hline Calles (Impermeable) & $12.00 \mathrm{E}-25$ & 0.1903 & 0.010 \\
\hline Lattosolo & $8.33 \mathrm{E}-8$ & 0.3163 & 0.385 \\
\hline Nittosolos Brunos & $1.77 \mathrm{E}-7$ & 0.3163 & 0.385 \\
\hline Nittosolos Litólicos & $1.77 \mathrm{E}-7$ & 0.3163 & 0.385 \\
\hline Cauces Permeables/Lagunas & $12.00 \mathrm{E}-0$ & 0.1903 & 1.00 \\
\hline Parámetros de rugosidad del modelo TREX. \\
\hline Usos del Suelo considerados & \multicolumn{3}{|c|}{ Rugosidad } \\
\hline [n de Manning] \\
\hline Vegetación arbórea & \multicolumn{3}{|c|}{0.300} \\
\hline Vegetación rastrera & \multicolumn{3}{|c|}{0.080} \\
\hline Suelo expuesto & \multicolumn{3}{|c|}{0.013} \\
\hline Áreas húmedas & \multicolumn{3}{|c|}{0.013} \\
\hline Construido & & \multicolumn{3}{|c|}{013} \\
\hline
\end{tabular}

Para la modelación de cada evento completo se tardó aproximadamente entre 2 a 5 días para modelar el escurrimiento en TREX dependiendo el evento y entre 2 a 4 horas para modelar el sistema hidráulico en SWMM, en una computadora con un procesador Intel Core i5-3230 M, con 8.00 GB de memoria RAM instalada

\section{CONCLUSIÓN}

A través del uso de los programas TREX y SWMM se logró realizar la calibración de un modelo totalmente distribuido en una cuenca fuertementeantropizada, pudiéndose simular elementos característicos de una cuenca urbana.

El procedimiento del trabajo en conjunto planteado entre un modelo totalmente distribuido como TREX y un modelo semi distribuido como SWMM, tuvo buenos resultados en la modelación de la cuenca urbana. Esto sigue la línea de las últimas investigaciones en modelación distribuida en áreas urbanas. Para la aplicación de este procedimiento, fue necesario la modificación parcial del código fuente del modelo TREX.

Los resultados de la calibración de ambos modelos funcionando en conjunto se pueden considerar aceptables teniendo en cuenta los valores que fueron reflejados en los indicadores de ajuste.

Por lo tanto, se logró obtener un modelo totalmente distribuido de la cuenca del Río Cascavel que refleja de manera aceptable su comportamiento y es posible obtener resultados en cada uno de los puntos de la cuenca, ya sea de escurrimientos superficiales, caudales en conductos, en cauces naturales, etc.

El trabajo permitió observar distintas dificultades que se presentan en la modelación hidrológica totalmente distribuida en una cuenca urbana, como por ejemplo: la incertidumbre que existe por la baja calidad de algunos de los datos de entrada del modelo, entre ellos: modelo digital de elevación, desconocimiento de ubicación, tamaño y disposición de los conductos de red de drenaje, datos de precipitación, etc. Por otro lado, se destaca el gran tiempo requerido para la simulación de cuencas urbanas al utilizar una resolución de 5 metros, tal cual se mencionó anteriormente, para cada evento completo se tardó aproximadamente entre 2 a 5 días para la modelación en TREX. Esta resolución fue elegida para las calles, construcciones y demás elementos del ámbito urbano.Por último, las modificaciones necesarias en los datos de ingreso, principalmente al modelo digital de elevación para poder representar lo más fielmente posible las diferencia de nivel de las calles con respecto al 
terreno natural, las parcelas y las barreras materializadas por construcciones o terraplenes.

A pesar de lo anterior, los modelos y el procedimiento establecido pueden ser utilizados sin inconvenientes en distintas cuencas urbanas ya que se contemplan todos los elementos que conforman a las mismas. Se deberá tener la precaución de evaluar el costo computacional según la extensión de la cuenca y resolución adoptada.

De esta forma, se puede concluir que se desarrolló un procedimiento de trabajo entre un modelo hidrológico distribuido (TREX) y un modelo semidistribuido (SWMM) el cual se utiliza su módulo de transporte hidráulico para simular de manera completa la transformación lluvia-caudal y el escurrimiento de una cuenca urbana.

La ventaja de este procedimiento es que utiliza las mejores características de ambos modelos. En primer lugar, tiene la capacidad de simular los escurrimientos superficiales con TREX a través de información brindada en formato raster, la cual en los últimos tiempos tiene un crecimiento sostenido en cuanto a calidad y disponibilidad. De esta forma, al obtener un MDE de buena calidad a través de relevamientos o información satelital se puede simular con TREX las direcciones de los escurrimientos de manera automática sin tener que indicar criterios aproximados como por ejemplo, tiempo de concentración, pendiente media, áreas de subcuecas, etc. Lo mismo sucede con el uso de suelo, a través de la información inferida de imágenes satelitales es fácilmente aplicable al modelo TREX para definir los parámetros de tipo y uso de suelo.

Por otro lado, se aprovecha las probadas potencialidades del modelo hidráulico que contiene SWMM modelando conductos abiertos y cerrados.

Las anteriores características de cada modelo se potencian al haber modificado el código fuente de TREX para que simule el flujo que ingresa al sistema de drenaje a través de las bocas de tormenta. De esta forma, se pudieron vincular ambos modelos utilizando las mejores características de cada uno.

\section{AGRADECIMIENTOS}

Los autores del presente trabajo quieren manifestar su agradecimiento al Conselho Nacional de
Desenvolvimento Científico e Tecnológico (CNPq) de Brasil por el apoyo económico, como así también a la Secretaría de Ciencia y Tecnología de la Universidad Tecnológica Nacional (Argentina).

\section{REFERENCIAS}

Abdullah, J., Muhammad, N. S., Muhammad, S. A., Mohammad Amin, N. F., y Tahir, W. (2019). Research trends in hydrological modelling. Jurnal Teknologi,81(4). https://doi.org/10.11113/jt.v81.13080

Armitage, N., Fisher-Jeffes, L., Carden, K., Winter, K., Naidoo, V., Spiegel, A., Mauck, B. y Coulson, D. (2014). Water Sensitive Urban Design (WSUD) for South Africa: Framework and Guidelines, WRC Report No. TT 588/14. Water Research Commission, Pretoria, South Africa.

Chow V.T., Maidment R. y Mays L. (1994). Hidrología Aplicada. Mc Graw-Hill.

Courty, L. G., Pedrozo-Acuña, A., y Bates, P. D.,(2017). Itzï (version 17.1): an open-source, distributed GIS model for dynamic flood simulation. Geoscientific Model Development, 10(4), 1835-1847.

England, J., Velleux, M. y Julien, P. (2007). Twodimensional simulations of extreme floods on a large watershed. Journal of Hydrology, 347(1), 229-241.

EPA (2005). SWMM - Manual del Usuario (Versión 2005, en español).

EPA (2015). SWMM - Storm Water Management ModelReference Manual - Volume I - Hidrology.

Freni, G, Maglionico, M. y Federico Di V. (2003). Estado del arte en la modelación de drenaje urbano. CARE-S Report D7.

Giangola-Murzyn, (2013). Modelación y parametrización hidrológica de la ciudad, resistencia a inundaciones. Tesis de Doctorado. Université Paris-Est.

Gomes, E. de S. (2014). A dinámica hidrológica fluvial em bacias hidrográficas com diferentes taxas de impermeabilizaçao do solo em Guarapuava/PR. Dissertaçao como requisito de Mestre em Geografia, UNICENTRO, Guarapuava, Parana.

Healy, R. W. (1990)Simulation of solute transport in variably saturated porous media with supplemental information on modifications to the U.S. GeologicalSurvey's Computer Program VS2D, U.S. Geological Survey Water Resources Investigations Report 90-4025, 125 p. 
INSA-Valor, S. O. G. R. E. A. H. (1999).Canoe: logiciel d'hydrologie urbaine, conception et évaluation de réseaux d'assainissement, simulation des pluies, des écoulements et de la qualité des eaux. Manuel de l'utilisateur.

Jorquera E., Weber J.F. y Reyna S.M. (2012). Revisión del estado del arte en la modelación hidrológica distribuida e integrada. Primer Encuentro de investigadores en formación en Recursos Hídricos. Ezeiza, Bs. As.

Julien, P. Y. y Saghafian, B. (1991). CASC2D user's manual: a two-dimensional watershed rainfall-runoff model. CER; 90/91-12.

Kleidorfer, M., Deletic, A., Fletcher, T. D. y Rauch, W. (2009). Impact of input data uncertainties on urban stormwater model parameters. Water Science and Technology, 60(6), 1545-1554.

Lind, J. (2015). Stormwater modelling tools: a comparison and evaluation.Tesis de Doctorado. Universidad de Uppsala, Suecia.

Mays, W. M. (2001). Stormwater Collection Systems Design Handbook. Mc Graw-Hill.

Mitchell, V. G., Duncan, H., Inman, M., Rahilly, M., Stewart, J., Vieritz, A., Holt, P., Grant, A., Fletcher, T., Coleman, J., Maheepala, S., Sharma, A., Deletic, A. y Breen, P. (2007).Integrated Urban Water Modelling - Past, Present, and Future. Joint 13th International Rainwater Catchment Systems Conference and the 5th International Water Sensitive Urban Design Conference, Conference 21-23 August, 2007, Sydney, Australia.

Mobayed, K. N. (2009). Simulador de inundaciones en áreas urbanas: Un modelo de pronóstico con fines de alertamiento. CIENCI@UAQ. 2, 72-90.

Mobayed K. N. y Cruickshank V. (1998). Ordenamiento sistemático de redes de drenaje para la modelación físico-hidrológica. In: XVIII Congreso Latinoamericano de Hidráulica. Oaxaca, México. pp: 611-620.

Mobayed K. N. y Ortiz, A. (2000). Modelo integral simplificado de las ecuaciones de Saint-Venant para el tránsito de avenidas. In: XIX Congreso Latinoamericano de Hidráulica. Córdoba, Argentina. 8 p.

Ochoa-Rodriguez, S., Wang, L-P., Assel J.V., Gires A., Ichiba A., Bruni G y ten Veldhuis M. C. (2013). Urban Pluvial Flood Modeling: Current Theory and Practice. Paris: RainGain. http://www.raingain.eu/sites/default/files/wp3_re view_document.pdf

Orsolini, H. E., Zimmermann, E.D. y Basile, P.A. (2000). Hidrología: Procesos y Métodos. ISBN
950-673- 254-4. UNR Editora. Editorial de la Universidad Nacional de Rosario.

Puricelli, M. M. (2003).Estimación y distribución de parámetros del suelo para la modelación hidrológica. Tesis Doctoral.Universidad Politécnica de Valencia, Departamento de Ingeniería Hidráulica y Medio Ambiente.

Rangari, V. A., Patel, A. K.y Umamahesh, N. V. (2015). Review of urban stormwater models. HYDRO 2015, 20th Int. Conf. On Hydraulics, IIT Roorkee, India.

Riccardi, G. (2004). Hidrología en medios antropizados. Libro electrónico. CURIHAM, FCEIA-UNR. http://cvirtual.fceia.unr.edu.ar.

Riccardi, G. A. (1997). La transformación lluviacaudal en ambientes rurales y urbanos. Los procesos hidrológicos y el modelado. Cuadernos del CURIHAM, 4, 69-87.

Rossman, L. A. (2010). Storm water management model user's manual, version 5.0 (p. 276). Cincinnati: National Risk Management Research Laboratory, Office of Research and Development, US Environmental Protection Agency.

Salvadore, E., Bronders, J., y Batelaan, O. (2015). Hydrological modelling of urbanized catchments: A review and future directions. Journal of hydrology, 529, 62-81

Sitterson, J., Knightes, C., Parmar, R., Wolfe, K., Muche, M. y Avant, B. (2017). An Overview of Rainfall-Runoff Model Types. U.S. Environmental Protection Agency, Washington, DC, EPA/600/R-17/482, 2017.

Stehli P.T., Weber J.F. y Jorquera E. (2012).Implementación de un modelo hidrológico espacialmente distribuido de simulación de eventos. Primer Encuentro de investigadores en formación en Recursos Hídricos. Ezeiza, Bs. As.

Stehli, P., Weber J. y Vestena L.(2016). Avances en la aplicación de un modelo hidrológico distribuido en una Cuenca fuertemente antropizada. XXVII Congreso Latinoamericano de Hidráulica 2016.

Stehli, P. T., Weber, J. F., y Vestena, L. R. (2021). Mapeamento dos bueiros na bacia do Rio Cascavel, cidade de Guarapuava, Brasil. Brazilian Journal of Development, 7(3), 30490-30508.

Tucci, C. E. (1998). Modelos hidrológicos. UFRGS.

Velleux, M., England, J. y Julien P. (2008). TREX: Spatially Distributed Model to Assess Watershed Contaminant Transport and Fate.Science of the Total Environment, 404(1):113-128.

Weber, J. F., Stehli, P. T., Jorquera, E. (2012). Simulación de la respuesta hidrológica de una 
cuenca ante el movimiento de una tormenta. Mecánica Computacional, XXXI(4), 579-603. Asociación Argentina de Mecánica Computacional, Salta. ISSN: 16666070.

Tipo de Publicación: ARTíCULO.

Trabajo recibido el 25/09/2021 y aprobado para su publicación el 29/10/2021.

\section{COMO CITAR}

Stehli, P. T., Weber, J. F. y Vestena, L. R. (2021). Aplicación y calibración de una integración de los modelos TREX y SWMM para la simulación hidrológica-hidráulica distribuida en una cuenca fuertemente antropizada. Cuadernos del CURIHAM. 27, 23-45.DOI: https://doi.org/10.35305/curiham.v27i.168

Este es un artículo de acceso abierto bajo licencia: Creative Commons Atribución - No Comercial Compartir Igual 4.0 Internacional (CC BY-NC-SA 4.0) (https://creativecommons.org/licenses/by-ncsa/4.0/deed.es) 